\title{
ANÁLISIS PRELIMINAR DE LA DOMINANCIA CULTURAL DE LAS PLANTAS ÚTILES EN EL ESTADO DE MORELOS
}

\author{
Columba Monroy-Ortiz' y Rafael Monroy ${ }^{2}$ \\ ${ }^{1}$ Laboratorio de Botánica Estructural, Centro de Investigación en Biotecnología (CelB) y \\ ${ }^{2}$ Laboratorio de Ecología, Centro de Investigaciones Biológicas (CIB). \\ Universidad Autónoma del Estado de Morelos, Av. Universidad 1001, Cuernavaca, 62210, Morelos, México. Tel: \\ (777) 3297000, ext. 3218 y 3219. Correo-e: columbam@cib.uaem.mx; ecologia@cib.uaem.mx.
}

\begin{abstract}
Resumen: Con base en la lista de plantas útiles en el estado de Morelos, recopilada de 1979 a 2001, se calculó la riqueza, la dominancia cultural y la frecuencia de uso de las familias, los géneros, las especies y los tipos de uso. Se registraron 608 especies pertenecientes a 400 géneros y 128 familias. Destacaron por su dominancia cultural las familias Fabaceae y Asteraceae, así como los géneros Ficus, Ipomoea y Citrus. Las 104 especies con mayor dominancia cultural se agruparon en 10 apartados con base en su origen y grado de manejo. Las especies dominantes culturalmente fueron Persea americana Mill. y Psidium guajava L. Se registraron 27 tipos de uso, siendo los dominantes el medicinal, el ornamental y el comestible. Se discute la relevancia del conocimiento tradicional en la selección de satisfactores vegetales y en la generación de ambientes que los promueven, a manera de contribución para el desarrollo sustentable.
\end{abstract}

Palabras clave: dominancia cultural, estado de Morelos, frecuencia de uso, plantas útiles, riqueza, tipo de uso.

\begin{abstract}
Richness, cultural dominance and use frequency of families, genera, species and use types of useful plants in the state of Morelos (Mexico) were evaluated. A database was built with the information gathered between 1979 and 2001 . A total of 608 species, 400 genera and 128 families were recorded. Fabaceae and Asteraceae were the culturally dominant families, whereas Ficus, Ipomoea and Citrus were the culturally dominant genera. According to origin and level of management, 10 groups of plants were defined that included the 104 culturally dominant species. The largest cultural dominance corresponded to Persea americana Mill. and Psidium guajava L. A total of 27 use types were recorded, among which medicinal, ornamental and edible were the culturally dominant ones. The importance of traditional knowledge in selecting useful products and its contribution to the creation of environments that promote the presence of these products is discussed, as well as the potential contribution that this kind of knowledge may make to sustainable development.
\end{abstract}

Keywords: cultural dominance, richness, state of Morelos, use frequency, use type, useful plants.

$\mathbf{L}$ os campesinos e indígenas de Mesoamérica forman unidades sociales de apropiación. Dichas unidades de tipo familiar y comunitario interactúan con la naturaleza a través de una estrategia no especializada de producción caracterizada por el uso múltiple de los recursos (Toledo y Argueta, 1994). Esta estrategia se fundamenta en el conocimiento tradicional, el cual a pesar de carecer de instrumentos de registro de información y de una metodología que le permita comparar y analizar los resultados con fines predictivos, cuenta con los mecanismos que le dan origen, lo conservan y lo transmiten de generación en generación, asegurando su continuidad a través del tiempo (Hernández-X., 1985).

Este tipo de conocimiento es una de las manifestaciones de la diversidad cultural de México, misma que es considera- da en los espacios de decisión, nacional e internacional, como de gran relevancia para la conservación ambiental (Convenio sobre Diversidad Biológica, 1992; Heywood, 1992; Ley General del Equilibrio Ecológico y Protección al Ambiente, 2003), así como para la elaboración de propuestas de manejo ambiental económicamente viables y ecológicamente factibles (Provencio y Carabias, 1993). El potencial del conocimiento tradicional supera el ámbito exclusivo de la conservación ambiental y proporciona elementos que pueden ser utilizados en la generación de un modelo de desarrollo nacionalista (Toledo, 1982) en el que la justicia social garantice el respeto a la naturaleza (Barrau, 1981).

Antes de incorporar al conocimiento tradicional en la estrategia de desarrollo nacional es necesaria su sistemati- 
zación, de modo que quede disponible para resolver las múltiples necesidades sociales (del Amo y Anaya, 1982; Gispert, 1992). Por ejemplo, en el caso de la atención a la salud se reconoce el aporte de la etnofarmacología como una vía para sistematizar el conocimiento etnomédico y hacerlo disponible para la generación de nuevos fármacos (Li et al., 2003). Como un valor adicional, el conocimiento etnomédico nacional podría protegerse legalmente e impulsarse para promover su participación en el mercado mundial de plantas medicinales. Este mercado fue valorado en 30 mil millones de dólares para el año 2000, con un incremento estimado de entre 5 y $15 \%$ anual (Vasisht y Kumar, 2002). A pesar de los beneficios que resultarían de combinar el conocimiento tradicional y el occidental en las propuestas de uso y manejo de los recursos, el primero se encuentra permanentemente amenazado por la desaparición. La erosión cultural avanza (Benz et al., 2000; Agelet y Valles, 2001) mientras que los esfuerzos por sistematizar el conocimiento tradicional y hacerlo disponible para su utilización no constituyen una prioridad en la estrategia de desarrollo nacional.

Existen diferentes propuestas para sistematizar cuantitativamente el conocimiento tradicional (Cervantes y Valdés, 1990; Martínez-Lirola et al., 1996; Phillips, 1996; Raja et al., 1997; Ali-Shtayeh et al., 2000; Said et al., 2002); entre otros aspectos, éstas incluyen los métodos para estimar la importancia cultural de las especies (Phillips y Gentry,
1993) y su nivel de uso (Muthcnick y McCarthy, 1997; Begossi et al., 2002). De manera complementaria a estas propuestas, basadas en el número de informantes que mencionan la utilidad de una planta, hay otras que promueven la utilización de los parámetros de la ecología para sistematizar al conocimiento tradicional (Rico-Gray, 1991; Figueiredo et al., 1993; Begossi, 1996; Figueiredo et al., 1997; Benz et al., 2000; Begossi et al., 2002).

El presente artículo contribuye a la identificación del potencial del conocimiento tradicional relacionado con las plantas útiles en el estado de Morelos, usando algunos parámetros de la ecología para su evaluación cuantitativa. El objetivo de este trabajo es sistematizar el conocimiento tradicional relacionado con la riqueza, la dominancia cultural y la frecuencia de uso de las familias, los géneros y las especies útiles, y de los tipos de uso dominantes culturalmente, así como reconocer las familias botánicas que dominan culturalmente en cada tipo de uso.

\section{Área de estudio}

El estado de Morelos está situado entre las coordenadas $18^{\circ} 20^{\prime}$ y $19^{\circ} 08^{\prime}$ de latitud N, y $98^{\circ} 38^{\prime}$ y $99^{\circ} 30^{\prime}$ de longitud $\mathrm{O}$ (figura 1). A pesar de su pequeña extensión territorial, equivalente a $0.2 \%$ de la superficie del país, Morelos posee una amplia variedad de ambientes determinados, entre otros factores, por la presencia de dos sistemas montañosos

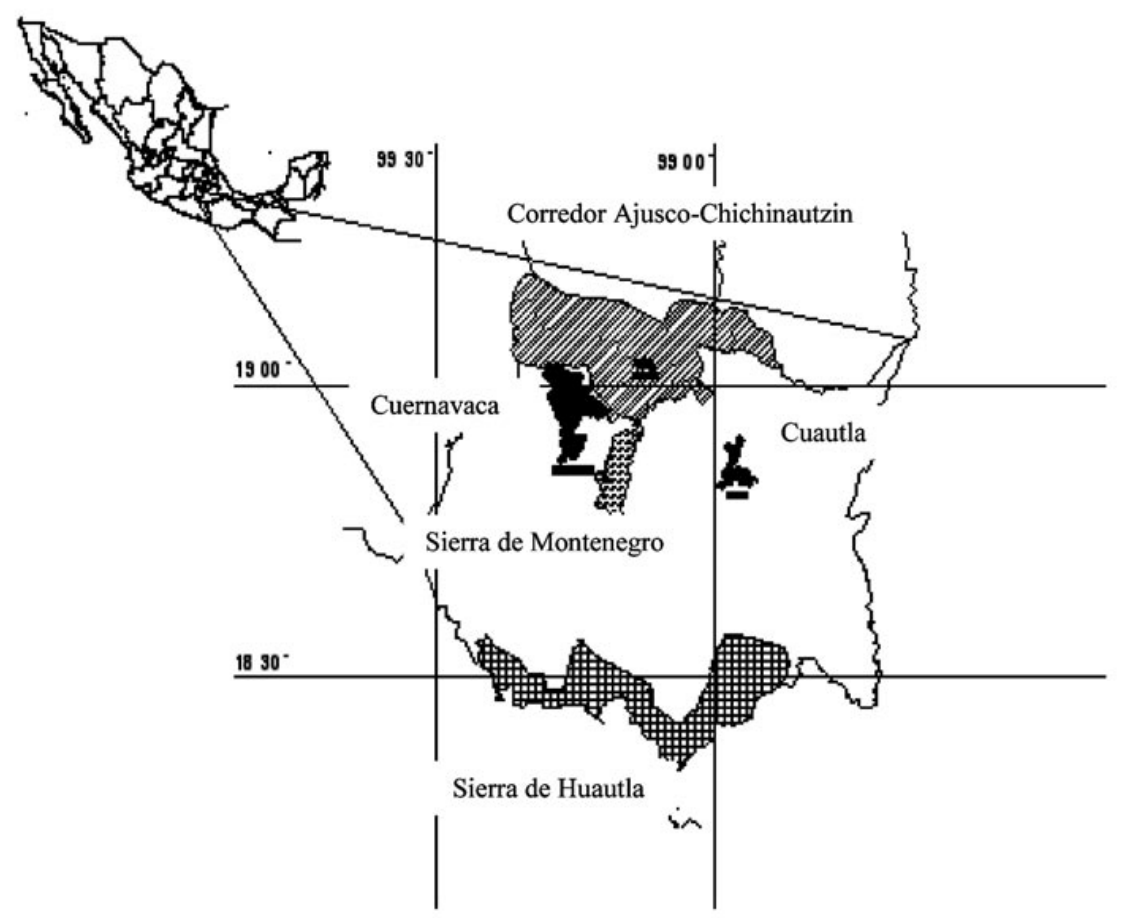

Figura 1. Ubicación del estado de Morelos. Se señalan las ciudades principales (Cuernavaca y Cuautla), así como las tres principales áreas naturales protegidas. 
que forman parte del Eje Neovolcánico Transversal y de la Sierra Madre del Sur. Esto permite regionalizar al estado por su clima y vegetación en tres zonas principales (Monroy et al., 1992).

La primera es la zona norte, donde se encuentra el Área de Protección de Flora y Fauna Silvestre "Corredor Biológico Ajusco-Chichinautzin" (37,302 ha), con climas templados y fríos, y cubierta por bosques de pino, pinoencino y oyamel. La segunda, la zona central, está formada por los valles de Cuernavaca y Cuautla, donde se realizan actividades agrícolas intensivas. Estos valles están separados entre sí por un pequeño sistema montañoso que conforma la Reserva Estatal "Sierra de Montenegro" (7,328 ha), área de distribución del bosque tropical caducifolio (Rzedowski, 1978) que se encuentra fuertemente amenazado por el crecimiento urbano de Cuernavaca y Cuautla. La tercera, zona sur, está caracterizada por un relieve montañoso que alberga la mayor superficie de bosque tropical caducifolio en el estado y en donde ha sido creada la Reserva de la Biosfera "Sierra de Huautla" (59,030 ha). En términos biológicos, es importante destacar la importancia de la ubicación de Morelos en una zona de convergencia de dos islas ecológicas muy importantes por su endemismo, el Eje Neovolcánico Transversal y la cuenca del río Balsas (Rzedowski, 1992b).

Morelos cuenta con antiguas raíces históricas, como lo demuestran los vestigios prehispánicos pertenecientes a la cultura olmeca encontrados en Chalcatzingo, los tlahuicas en Xochicalco y los xochimilcas en Tepoztlán (Maldonado, 1990). Otros acontecimientos históricos que han influido en el devenir de la cultura y el ambiente morelense han sido la presencia de la hacienda azucarera (Von Wobeser, 1989) y el surgimiento del zapatismo durante la Revolución Mexicana de 1910. Es así como la cultura morelense se ha construido con elementos propios y con los provenientes de otras regiones (América, Europa, África y Asia) a través del intercambio económico y cultural, o bien por la imposición por parte de la cultura dominante en turno. En consecuencia, la interacción de la sociedad caracterizada por su diversidad cultural con un ambiente igualmente diverso ha influido en la generación del conocimiento que sustenta el uso y el manejo de los recursos vegetales en Morelos.

\section{Materiales y métodos}

El conocimiento que aquí se sistematiza es resultado del trabajo del Laboratorio de Ecología del Centro de Investigaciones Biológicas (CIB), Universidad Autónoma del Estado de Morelos (UAEM), realizado entre 1979 y 2001 (cuadro 1). Estos estudios, realizados en alguna o en las tres principales zonas ambientales de Morelos, han empleado diferentes enfoques y escalas espaciales para generar información sobre el uso y manejo tradicional de los recursos vegetales. A partir de estas fuentes, se obtuvo la información relacionada con las familias, los géneros y las especies usadas, así como los tipos de uso de los recur-

Cuadro 1. Enfoques empleados por los diferentes colaboradores del Laboratorio de Ecología, entre 1979 y 2001.

\begin{tabular}{|c|c|c|c|}
\hline \multirow[t]{2}{*}{ Enfoque } & \multicolumn{3}{|c|}{ Zona } \\
\hline & Norte & Central & Sur \\
\hline Inventarios de plantas útiles & $\begin{array}{l}\text { Hernández, 1983*; } \\
\text { Matosic, 1991* }\end{array}$ & Ayala, 1998* & Pérez, 1982* \\
\hline Plantas comestibles & $\longleftarrow$ & Taboada, $1981^{* * *}$ & $\overrightarrow{\text { Maldonado y Monroy, 1989a** }}$ \\
\hline Plantas medicinales & Sánchez, 1982* & $\begin{array}{c}\text { Chávez, 1979*; Villegas, 1979*; } \\
\text { Bajonero, 1982* }\end{array}$ & \\
\hline Plantas usadas para elaborar artesanías & $\longleftarrow$ & Aguirre, $1993^{* * *}$ & $\longrightarrow$ \\
\hline Plantas ornamentales & & $\begin{array}{c}\text { Flores, } 1988^{*} ; \\
\text { Altamirano, 2001* }\end{array}$ & \\
\hline Plantas usadas para la construcción & & & Maldonado y Monroy, 1989b** \\
\hline Huertos familiares & Colín, 1990* & Aguilar, 1993*; Salazar, 1994* & Antonio, $1997^{*}$ \\
\hline Sistemática vegetal & $\longleftarrow$ & Martínez, 1985*** & $\longrightarrow$ \\
\hline Etnoecología & Bello y Vilchis, 1989* & & Monroy-Ortiz, 1997* \\
\hline Bromatología y propagación & & Villa, 1993* & \\
\hline Educación ambiental y plantas útiles & & Quezada, 1990* & \\
\hline Plantas comercializadas en mercado & & Monroy et al., 1986* & \\
\hline
\end{tabular}

* estudio local, ** estudio regional (incluye más de un municipio), *** estudio estatal 
sos vegetales registrados en Morelos.

La información botánica fue revisada con el fin de eliminar las sinonimias y corregir el nombre de los autores de las especies. Los nombres científicos se ajustaron de acuerdo a diferentes floras (del Valle de México, del Bajío y de Regiones Adyacentes, de Veracruz, de Guatemala, Mesoamericana, Neotropica) y con la base de datos VAST del Missouri Botanical Garden (2002). Se anotó la región de origen de las especies con el fin de poder analizar su dominancia cultural; cuando esto no fue posible debido a la falta de información, se registró el área de distribución.

Los autores se abreviaron de acuerdo a Brummitt y Powell (1992); las familias se clasificaron con base en Davidse et al. (1995) (Pteridophyta), Espinosa (2001) (Gymnospermae), Dahlgren et al. (1985) (Monocotyledoneae) y Cronquist (1981) (Dycotiledoneae).

La información recabada sobre el conocimiento tradicional vinculado con el uso de los recursos vegetales en Morelos fue evaluada cuantitativamente por medio del cálculo de algunos índices desarrollados por la ecología de comunidades, previamente modificados conforme a la perspectiva etnobotánica. El empleo de parámetros ecológicos para realizar una evaluación cuantitativa del conocimiento tradicional referente al uso de los recursos naturales ha sido propuesto con anterioridad. Por ejemplo, se han usado índices de diversidad (Rico-Gray, 1991; Begossi, 1996; Figueiredo et al., 1997; Benz et al., 2000; Begossi et al., 2002) y elementos de la teoría de biogeografía de islas (Figueiredo et al., 1993) para explicar los cambios en el conocimiento sobre el uso de los recursos vegetales. En este estudio se propone el empleo de la riqueza, la dominancia y la frecuencia de uso, con las modificaciones que se explican a continuación.

Riqueza de las plantas útiles en el estado de Morelos. En ecología, la riqueza equivale al número de especies (Krebs, 1985), géneros o familias registradas en un lugar de muestreo. Aquí se cuantificó la riqueza en cada una de estas categorías taxonómicas a partir de la información obtenida con las plantas útiles en Morelos. Además, para evaluar la disponibilidad de recursos vegetales usados en el estado, se comparó la riqueza registrada en este trabajo con la citada en la flora de Morelos (Bonilla-Barbosa y Villaseñor, 2003) y en la base nacional de información sobre plantas con uso (Caballero y Cortés, 2001).

Se estimó el número de especies por km² (Agelet y Valles, 2001), parámetro conocido como riqueza relativa (Casas et al., 2001), con el fin de comparar nuestros resultados con los obtenidos para regiones con diferentes culturas y ambientes, considerando que ésta es una de las vías para identificar patrones explicativos generalizables sobre esta interacción (Moerman et al., 1999). Este procedimiento es complementario en la evaluación de la disponibilidad de satisfactores obtenidos a partir de plantas.
Dominancia cultural de las plantas útiles en el estado de Morelos. La dominancia expresada en términos absolutos o relativos es un parámetro utilizado para expresar el grado en que una especie acapara el espacio de crecimiento en una comunidad (Krebs, 1985). En este trabajo la dominancia cultural muestra la relevancia diferencial de las familias, los géneros y las especies como fuentes de satisfactores de las múltiples necesidades sociales. A continuación se detallan los diferentes tipos de dominancia que se calcularon en este trabajo.

Dominancia cultural de las familias basada en el número de especies. - Se calculó la dominancia cultural absoluta anotando el número de especies registradas en cada familia, y la dominancia cultural relativa resultó de la estimación de la distribución proporcional de dichas especies en las diferentes familias (Monroy-Ortiz y Monroy, 2001). Estos valores relativos sirven para estandarizar, haciendo posible la evaluación comparativa de los recursos vegetales usados en Morelos.

Dominancia cultural de las familias basada en el número de géneros.- La dominancia cultural absoluta está representada por el número de géneros registrados en cada familia, mientras que la dominancia cultural relativa equivale a su distribución proporcional.

Dominancia cultural de los géneros basada en el número de especies. - Se calcularon dos tipos de dominancia cultural, la absoluta y la relativa. La primera representa el número de especies registradas en cada género y la segunda es igual a la representación proporcional de las mismas.

Frecuencia de uso. En estudios ecológicos, el análisis de la distribución de frecuencias de diámetros o alturas complementa la valoración de la repartición del espacio de crecimiento en una comunidad. En este caso, se esperaba obtener una medida complementaria sobre la forma en la que se distribuye la satisfacción de necesidades a partir de diferentes familias y géneros. Se presenta la distribución de especies y familias con respecto al tamaño de la familia (número de especies), así como la distribución de géneros y especies en función de la riqueza de especies por cada género.

Dominancia cultural de las plantas útiles en Morelos. El número de citas que recibe una planta ha sido empleado para estimar la importancia cultural de las especies (Phillips y Gentry, 1993), así como su nivel de uso (Muthcnick y McCarthy, 1997; Begossi et al., 2002). Además, ha servido como una medida de la efectividad (Ali-Shtayeh et al., 2000) y confiabilidad (Raja et al., 1997; Said et al., 2002) del conocimiento tradicional, de la distribución de su utilización (Martínez-Lirola et al., 1996) 
y de sus niveles de arraigo cultural en la población (Cervantes y Valdés, 1990). En el presente estudio, se coincide con quienes plantean que el número de citas proporciona una medida de la intensidad del uso y de la amplitud en la distribución del conocimiento sobre el uso de los recursos vegetales. Esta medida recibe el nombre de dominancia cultural absoluta y se cuantifica a partir del número de estudios, en este caso los 24 realizados en el Laboratorio de Ecología, en los que se cita una especie. También se calculó la dominancia cultural relativa, utilizando para ello la proporción de citas que ha recibido cada especie con respecto al total (24).

Dominancia cultural de los tipos de uso. Se enlistaron los diferentes tipos de uso señalados en la bibliografía consultada, anotando el número de especies correspondientes a cada uno para obtener así su dominancia cultural absoluta. La dominancia cultural relativa se obtuvo a partir de la estimación de la distribución proporcional de las especies en los diferentes tipos de uso. Algunas especies tienen más de un tipo de uso y por lo tanto la suma de las proporciones no es igual a 100 puntos. Además, se estimó la dominancia cultural, tanto absoluta como relativa, de las familias botánicas en las diferentes categorías de uso.

\section{Resultados}

Riqueza de las plantas útiles en el estado de Morelos. Se registraron en total 608 especies vegetales con algún tipo de uso, pertenecientes a 400 géneros y 128 familias (cuadro 2). Destacan por su riqueza las espermatofitas con 604 especies (99.3\%), las angiospermas con $595(97.9 \%)$ y las dicotiledóneas con 528 (86.8\%).

En Morelos se han registrado hasta el momento 3,686 especies de plantas vasculares (Bonilla-Barbosa y Villaseñor, 2003), de manera que las especies útiles representan $16.5 \%$ (608) de la flora de Morelos. Por lo que respecta a los géneros, en el estado hay inventariados
1,016, de los cuales $39.4 \%$ tienen algún uso. Asimismo, $64.7 \%$ (128 de 198) de las familias poseen especies útiles.

Se ha estimado que en México existen 7,000 especies útiles (Caballero y Cortés, 2001), así que las 608 mencionadas en este documento representan $8.8 \%$ de las mismas. Si la riqueza de especies útiles fuera sólo consecuencia de la extensión territorial, en México (1,964,375 km²) se tendrían alrededor de 0.0036 especies útiles $\mathrm{km}^{-2}$ mientras que en Morelos, con sólo $0.25 \%$ de la superficie nacional $\left(4,958 \mathrm{~km}^{2}\right)$, se esperaría una cifra muchas veces menor que la calculada, que es de 0.12 especies $\mathrm{km}^{-2}$. Esta riqueza relativa también fue mayor en Morelos que la señalada por Casas et al. (2001) para Tehuacán (0.08), la Sierra Norte de Puebla (0.06), la región de Uxpanapa (0.07), la Selva Lacandona (0.03), la Reserva de la Biosfera de Sian Ka'an (0.06), la zona tepehuana (0.04), la Península de Yucatán (0.01) y la región de La Montaña (Guerrero; 0.04). El único lugar que tiene un índice 3.5 veces mayor que el de Morelos es Manantlán, donde para una superficie de $1,400 \mathrm{~km}^{2}$ se han registrado 650 especies útiles, obteniéndose en consecuencia una riqueza relativa de 0.46 especies útiles $\mathrm{km}^{-2}$ (Benz et al., 2000).

Dominancia cultural de las plantas útiles en el estado de Morelos. Dominancia cultural de las familias basada en el número de especies (cuadro 3). - De las 128 familias con especies útiles destacan Fabaceae, con una dominancia absoluta de 58 especies y relativa de $9.5 \%$ (cuadro 4), Asteraceae con $48(7.9 \%)$, así como Lamiaceae y Solanaceae con 21 (3.5\%), seguidas por Cactaceae con 17 especies $(2.8 \%)$, Arecaceae, Euphorbiaceae y Poaceae con $16(2.6 \%)$.

Dominancia cultural de las familias con base en el número de géneros. - Las familias con especies útiles que dominan en Morelos son (cuadro 5): Fabaceae con una dominancia absoluta de 32 géneros y una dominancia relativa de $8.0 \%$, Asteraceae con 30 (7.5\%), Poaceae con 14 (3.5\%),

Cuadro 2. Riqueza de las plantas útiles en el estado de Morelos.

\begin{tabular}{|c|c|c|c|c|c|c|}
\hline & \multicolumn{2}{|c|}{ Especies } & \multicolumn{2}{|c|}{ Géneros } & \multicolumn{2}{|c|}{ Familias } \\
\hline & No. & $\%$ & No. & $\%$ & No. & $\%$ \\
\hline Pteridophyta & 4 & 0.66 & 4 & 1.00 & 4 & 3.13 \\
\hline Spermatophyta & 604 & 99.34 & 396 & 99.00 & 124 & 96.88 \\
\hline Gymnospermae & 13 & 2.14 & 7 & 1.75 & 4 & 3.13 \\
\hline Angiospermae & 595 & 97.86 & 393 & 98.25 & 124 & 96.88 \\
\hline Monocotyledoneae & 80 & 13.16 & 58 & 1.45 & 20 & 15.63 \\
\hline Dicotyledoneae & 528 & 86.84 & 342 & 85.50 & 108 & 84.38 \\
\hline Total & 608 & & 400 & & 128 & \\
\hline
\end{tabular}




\section{Columba Monroy-Ortiz y Rafael Monroy}

Cuadro 3. Comparación de la dominancia cultural de las familias de la flora de Morelos con las que poseen especies útiles en Morelos y México.

\begin{tabular}{|c|c|c|c|c|c|c|c|c|c|}
\hline \multirow[b]{2}{*}{ Familias } & \multicolumn{3}{|c|}{ Flora de Morelos* } & \multicolumn{3}{|c|}{ Útiles en Morelos } & \multicolumn{3}{|c|}{ Útiles en México*** } \\
\hline & $\begin{array}{c}\text { Dominancia } \\
\text { cultural } \\
\text { absoluta } \\
\text { (No. de especies) }\end{array}$ & $\begin{array}{c}\text { Dominancia } \\
\text { cultural } \\
\text { relativa } \\
(\%) \\
\end{array}$ & $\begin{array}{c}\text { Posición } \\
\text { por } \\
\text { dominancia } \\
\text { cultural } \\
\end{array}$ & $\begin{array}{c}\text { Dominancia } \\
\text { cultural } \\
\text { absoluta } \\
\text { (No. de especies) }\end{array}$ & $\begin{array}{c}\text { Dominancia } \\
\text { cultural } \\
\text { relativa } \\
(\%) \\
\end{array}$ & $\begin{array}{c}\text { Posición } \\
\text { por } \\
\text { dominancia } \\
\text { cultural } \\
\end{array}$ & $\begin{array}{c}\text { Dominancia } \\
\text { cultural } \\
\text { absoluta } \\
\text { (No. de especies) }\end{array}$ & $\begin{array}{c}\text { Dominancia } \\
\text { cultural } \\
\text { relativa } \\
(\%) \\
\end{array}$ & $\begin{array}{c}\text { Posición } \\
\text { por } \\
\text { dominancia } \\
\text { cultural } \\
\end{array}$ \\
\hline Fabaceae & $* * 293$ & 7.95 & 2 & 58 & 9.54 & 1 & 299 & 8.54 & 2 \\
\hline Asteraceae & 447 & 12.13 & 1 & 48 & 7.89 & 2 & 304 & 8.69 & 1 \\
\hline Lamiaceae & 75 & 2.03 & 8 & 21 & 3.45 & 3 & 101 & 2.89 & 6 \\
\hline Solanaceae & 82 & 2.22 & 7 & 21 & 3.45 & 4 & 117 & 3.34 & 3 \\
\hline Euphorbiaceae & 91 & 2.47 & 5 & 16 & 2.63 & 7 & 115 & 3.29 & 4 \\
\hline Poaceae & 216 & 5.86 & 3 & 16 & 2.63 & 8 & - & - & - \\
\hline Cactaceae & - & - & - & 17 & 2.80 & 5 & 110 & 3.14 & 5 \\
\hline Rosaceae & - & - & - & 15 & 2.47 & 9 & 59 & 1.69 & 10 \\
\hline Rutaceae & - & - & - & 15 & 2.47 & 10 & 45 & 1.29 & 11 \\
\hline
\end{tabular}

* Bonilla-Barbosa y Villaseñor (2003); ** Fabaceae+Mimosaceae; ${ }^{* * *}$ Caballero y Cortés (2001).

Cuadro 4. Dominancia cultural, absoluta y relativa, de las familias, con base en el número de especies.

\begin{tabular}{|c|c|c|}
\hline \multirow[t]{2}{*}{ Familia } & \multicolumn{2}{|c|}{ Dominancia cultural } \\
\hline & $\begin{array}{c}\text { Absoluta } \\
\text { (No. de especies) }\end{array}$ & $\begin{array}{c}\text { Relativa } \\
(\%)\end{array}$ \\
\hline$\overline{\text { Fabaceae }}$ & 58 & 9.54 \\
\hline Asteraceae & 48 & 7.89 \\
\hline Lamiaceae & 21 & 3.45 \\
\hline Solanaceae & 21 & 3.45 \\
\hline Cactaceae & 17 & 2.80 \\
\hline Arecaceae & 16 & 2.63 \\
\hline Euphorbiaceae & 16 & 2.63 \\
\hline Poaceae & 16 & 2.63 \\
\hline Rosaceae & 15 & 2.47 \\
\hline Rutaceae & 15 & 2.47 \\
\hline Moraceae & 14 & 2.30 \\
\hline Bignoniaceae & 12 & 1.97 \\
\hline Apocynaceae & 11 & 1.81 \\
\hline Convolvulaceae & 10 & 1.64 \\
\hline Myrtaceae & 10 & 1.64 \\
\hline Verbenaceae & 10 & 1.64 \\
\hline Anacardiaceae & 9 & 1.48 \\
\hline Araceae & 9 & 1.48 \\
\hline Boraginaceae & 9 & 1.48 \\
\hline Cucurbitaceae & 8 & 1.32 \\
\hline Malvaceae & 8 & 1.32 \\
\hline Apiaceae & 7 & 1.15 \\
\hline Burseraceae & 7 & 1.15 \\
\hline Agavaceae & 6 & 0.99 \\
\hline Annonaceae & 6 & 0.99 \\
\hline Brassicaceae & 6 & 0.99 \\
\hline Chenopodiaceae & 6 & 0.99 \\
\hline Meliaceae & 6 & 0.99 \\
\hline Pinaceae & 6 & 0.99 \\
\hline Acanthaceae & 5 & 0.82 \\
\hline Amaranthaceae & 5 & 0.82 \\
\hline Commelinaceae & 5 & 0.82 \\
\hline Fagaceae & 5 & 0.82 \\
\hline Geraniaceae & 5 & 0.82 \\
\hline Oleaceae & 5 & 0.82 \\
\hline Rubiaceae & 5 & 0.82 \\
\hline Sapotaceae & 5 & 0.82 \\
\hline
\end{tabular}

Cuadro 5. Dominancia cultural absoluta y relativa de las familias con base en el número de géneros.

\begin{tabular}{|c|c|c|}
\hline \multirow[t]{2}{*}{ Familias } & \multicolumn{2}{|c|}{ Dominancia cultural } \\
\hline & $\begin{array}{c}\text { Absoluta } \\
\text { (No. de géneros) }\end{array}$ & $\begin{array}{c}\text { Relativa } \\
(\%)\end{array}$ \\
\hline Fabaceae & 32 & 8.00 \\
\hline Asteraceae & 30 & 7.50 \\
\hline Poaceae & 14 & 3.50 \\
\hline Arecaceae & 13 & 3.25 \\
\hline Lamiaceae & 13 & 3.25 \\
\hline Cactaceae & 12 & 3.00 \\
\hline Euphorbiaceae & 10 & 2.50 \\
\hline Rosaceae & 10 & 2.50 \\
\hline Bignoniaceae & 9 & 2.25 \\
\hline Solanaceae & 9 & 2.25 \\
\hline Apiaceae & 7 & 1.75 \\
\hline Araceae & 7 & 1.75 \\
\hline Malvaceae & 7 & 1.75 \\
\hline Myrtaceae & 7 & 1.75 \\
\hline Verbenaceae & 7 & 1.75 \\
\hline Anacardiaceae & 6 & 1.50 \\
\hline Apocynaceae & 6 & 1.50 \\
\hline Boraginaceae & 6 & 1.50 \\
\hline Rutaceae & 6 & 1.50 \\
\hline Acanthaceae & 5 & 1.25 \\
\hline Cucurbitaceae & 5 & 1.25 \\
\hline Rubiaceae & 5 & 1.25 \\
\hline Amaranthaceae & 4 & 1.00 \\
\hline Brassicaceae & 4 & 1.00 \\
\hline Ericaceae & 4 & 1.00 \\
\hline Lythraceae & 4 & 1.00 \\
\hline Malpighiaceae & 4 & 1.00 \\
\hline Meliaceae & 4 & 1.00 \\
\hline Moraceae & 4 & 1.00 \\
\hline Sapotaceae & 4 & 1.00 \\
\hline Sterculiaceae & 4 & 1.00 \\
\hline Araliaceae & 3 & 0.75 \\
\hline Bombacaceae & 3 & 0.75 \\
\hline Cupressaceae & 3 & 0.75 \\
\hline Chenopodiaceae & 3 & 0.75 \\
\hline Lauraceae & 3 & 0.75 \\
\hline Nyctaginaceae & 3 & 0.75 \\
\hline Oleaceae & 3 & 0.75 \\
\hline Scrophulariaceae & 3 & 0.75 \\
\hline
\end{tabular}


Cuadro 6. Comparación de la dominancia cultural de las familias con especies útiles y de la flora de Morelos, con base en su número de géneros.

\begin{tabular}{|c|c|c|c|c|c|c|}
\hline & \multicolumn{3}{|c|}{ Útiles de Morelos } & \multicolumn{3}{|c|}{ Flora de Morelos } \\
\hline & $\begin{array}{c}\text { Dominancia } \\
\text { cultural absoluta } \\
\text { (No. de géneros) }\end{array}$ & $\begin{array}{c}\text { Dominancia } \\
\text { cultural } \\
\text { relativa }(\%)\end{array}$ & $\begin{array}{c}\text { Posición por } \\
\text { dominancia } \\
\text { cultural }\end{array}$ & $\begin{array}{c}\text { Dominancia } \\
\text { cultural absoluta } \\
\text { (No. de géneros) }\end{array}$ & $\begin{array}{l}\text { Dominancia } \\
\text { cultural } \\
\text { relativa }(\%)\end{array}$ & $\begin{array}{c}\text { Posición por } \\
\text { dominancia } \\
\text { cultural }\end{array}$ \\
\hline Fabaceae & 32 & 8.00 & 1 & 58 & 5.71 & 2 \\
\hline Asteraceae & 30 & 7.50 & 2 & 127 & 12.50 & 1 \\
\hline Poaceae & 14 & 3.50 & 3 & 68 & 6.69 & 3 \\
\hline Arecaceae & 13 & 3.25 & 4 & - & - & - \\
\hline Lamiaceae & 13 & 3.25 & 5 & 12 & 1.18 & 8 \\
\hline Cactaceae & 12 & 3.00 & 6 & - & - & - \\
\hline Euphorbiaceae & 10 & 2.50 & 7 & 19 & 1.87 & 5 \\
\hline Rosaceae & 10 & 2.50 & 8 & - & - & - \\
\hline Bignoniaceae & 9 & 2.25 & 9 & - & - & - \\
\hline Solanaceae & 9 & 2.25 & 10 & 14 & 1.38 & 7 \\
\hline
\end{tabular}

Arecaceae y Lamiaceae con $13(3.3 \%)$. Seis de las 10 familias dominantes por su número de géneros en la flora de Morelos también se encuentran en la lista de las familias que destacan por su número de especies útiles e incluyen: Fabaceae, Asteraceae, Poaceae, Lamiaceae, Euphorbiaceae y Solanaceae (cuadro 6).

Dominancia cultural de los géneros obtenida con base en el número de especies. - Los géneros de las plantas útiles dominantes culturalmente en Morelos son (cuadro 7): Ficus, con una dominancia absoluta de 11 especies y una relativa de $1.8 \%$, Ipomoea y Citrus, con 9 (1.5\%), Acacia, con $8(1.3 \%)$, Bursera, con 7 (1.2\%). En seguida se tiene un grupo de cuatro géneros que comparten una dominancia absoluta de seis especies (1.0\%), el cual incluye a Annona, Euphorbia, Salvia y Solanum.

Distribución de frecuencias. El 53.9\% de las familias aportan únicamente una o dos especies útiles. Esta contribución disminuye de manera pronunciada conforme aumenta el número de especies por familia. Por otro lado, las especies se distribuyen con un patrón bastante irregular (figura 2).

En el caso de los géneros, $90.3 \%$ poseen sólo una o dos especies útiles, de acuerdo con un patrón de distribución inversamente proporcional. Asimismo, 70\% de las especies pertenece a géneros con una o dos especies útiles, conforme a una distribución similar a la citada anteriormente (figura 3).

Dominancia cultural de las plantas útiles en Morelos. En este apartado solamente se mencionan las 104 especies que reciben cuatro citas o más, debido a que su empleo con mayor intensidad (Muthcnick y McCarthy, 1997; Begossi et al., 2002) permite suponer una mayor contribución a la satisfacción de las necesidades sociales en Morelos.
Cuadro 7. Dominancia cultural de los géneros con base en su número de especies útiles en Morelos

\begin{tabular}{lcc}
\hline Géneros & \multicolumn{2}{c}{ Dominancia cultural } \\
\cline { 2 - 3 } & $\begin{array}{c}\text { Absoluta } \\
\text { (No. de especies) }\end{array}$ & $\begin{array}{c}\text { Relativa } \\
\text { (\% especies) }\end{array}$ \\
\hline Ficus & 11 & 1.81 \\
Ipomoea & 9 & 1.48 \\
Citrus & 9 & 1.48 \\
Acacia & 8 & 1.32 \\
Bursera & 7 & 1.15 \\
Annona & 6 & 0.99 \\
Euphorbia & 6 & 0.99 \\
Salvia & 6 & 0.99 \\
Solanum & 6 & 0.99 \\
Pinus & 5 & 0.82 \\
Porophyllum & 5 & 0.82 \\
Quercus & 5 & 0.82 \\
Senna & 5 & 0.82 \\
Agave & 4 & 0.66 \\
Artemisia & 4 & 0.66 \\
Brugmansia & 4 & 0.66 \\
Chenopodium & 4 & 0.66 \\
Leucaena & 4 & 0.66 \\
Plumeria & 4 & 0.66 \\
Prunus & 4 & 0.66 \\
Stenocereus & 4 & 0.66 \\
Tagetes & 4 & 0.66 \\
\hline & 4 &
\end{tabular}

Además, se supone que el conocimiento tradicional vinculado al uso de este grupo de especies se encuentra distribuido más ampliamente (apéndice 2).

Las especies con mayor dominancia cultural son Persea americana y Psidium guajava con 13 citas (50.0\% de dominancia cultural relativa), seguidas por Pithecellobium dulce y Citrus aurantifolia con 12 (46.2\%). A continuación, con 


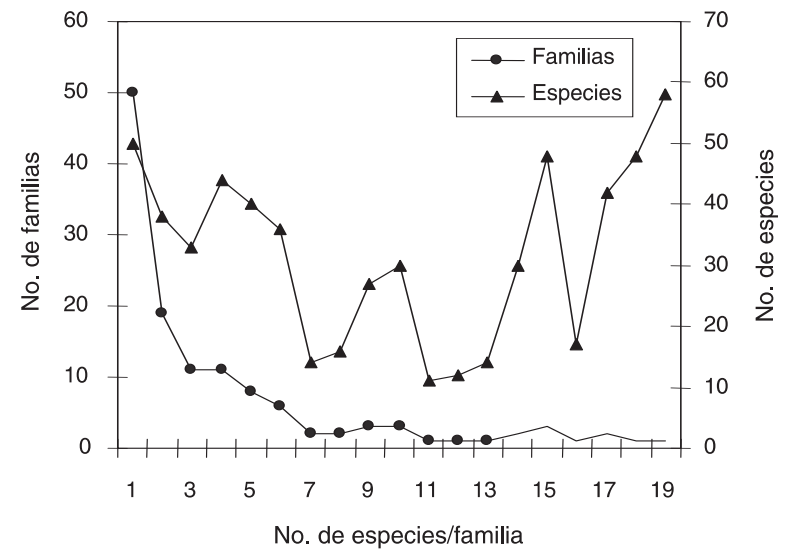

Figura 2. Distribución de frecuencias de las familias y las especies con respecto al número de especies registradas por familia.

11 citas (42.3\%) se tiene a Senecio salignus, Crescentia alata, Chenopodium ambrosioides y Ruta chalepensis.

En términos generales, las 104 plantas útiles dominantes culturalmente en Morelos (apéndice 2) se pueden clasificar, con base en su origen y grado de manejo, en los 10 grupos que se mencionan a continuación (cuadro 8). (1) Introducidas cultivadas, con 39 especies (37.5\%), incluye entre otras, al limón (Citrus aurantifolia) y la ruda (Ruta chalepensis). (2) Introducidas cultivadas y naturalizadas, con tres especies (2.9\%); incluye al tabachín (Delonix regia) y al tamarindo (Tamarindus indica). (3) Introducidas naturalizadas, con cinco especies (4.8\%); incluye al cuahulote (Guazuma ulmifolia) y la higuerilla (Ricinus communis). (4) Nativas cultivadas, con siete especies (6.7\%); incluye al maiz (Zea mays) y al muicle (Jacobinia spicigera). (5) Nativas cultivadas que crecen en zonas perturbadas, con cinco especies (4.8\%); incluye al epazote (Chenopodium ambrosioides) y al colorín (Erythrina americana). (6) Nativas cultivadas que crecen tanto en zonas perturbadas como relativamente conservadas, con dos especies (1.9\%); incluye al guaje (Leucaena esculenta) y al capulín (Prunus serotina ssp. capuli). (7) Nativas que crecen exclusivamente en zonas perturbadas, con 16 especies (15.4\%); incluye al guamúchil (Pithecellobium dulce) y al cuatecomate (Crescentia alata). (8) Nativas que crecen en zonas relativamente conservadas, con ocho especies (7.7\%); incluye al tlahuitol (Lysiloma divaricatum) y al guachocote (Malpighia mexicana). (9) Nativas cultivadas que crecen en zonas relativamente conservadas, con 11 especies $(10.6 \%)$; incluye la papaya (Carica papaya) y la ciruela (Spondias purpurea). (10) Nativas que crecen en zonas relativamente conservadas y perturbadas, con ocho especies (7.7\%), incluye al cuachalalate (Amphipterygium adstringens) y al cuajilote (Parmentiera aculeata).
De las especies útiles en Morelos que dominan culturalmente, $54.8 \%$ (57) se distribuyen en o son originarias de América y $7.7 \%$ (8) son nativas de Sudamérica, Cuba o las Antillas (cuadro 8). El 37.5\% (39) restante corresponde a especies introducidas de otras partes del mundo, entre las que destaca el origen asiático con 18 especies (17.3\%).

De acuerdo con el grado de manejo, 46 especies (44.2\%) se cultivan, $16(15.4 \%)$ crecen en zonas perturbadas socialmente, como la orilla de los caminos o en campos de cultivo en descanso, $11(10.6 \%)$ se encuentran silvestres y cultivadas, mientras que ocho $(7.7 \%)$ se desarrollan en ambientes silvestres relativamente conservados (cuadro 8).

Las 104 especies con una dominancia de cuatro citas o más pertenecen a 45 familias, 53.3\% de las cuales (24) tienen una sola especie. Se distribuyen en 89 géneros; $89.9 \%$ (80) de éstos sólo aportan una especie. Los géneros con un mayor número de especies son Citrus con siete $(6.7 \%)$ y Mentha con tres (2.9\%) (apéndice 2).

Por lo que se refiere a las formas de vida, sobresale la arbórea con 57 especies (54.8\%), seguida por la arbustiva con $14(13.5 \%)$ y la herbácea con 31 (29.8\%).

Dominancia cultural de los diferentes tipos de uso. Se registraron 27 tipos de uso (cuadro 9), entre los que destaca el uso medicinal con una dominancia absoluta de 293 especies o $48.2 \%$ de dominancia relativa, 12 puntos menos que en el registro nacional (61.1\%; Caballero y Cortés, 2001). En seguida se tiene al ornamental con 255 (41.9\%) y al comestible con 169 (27.8\%); este último valor fue similar al de $27.1 \%$ registrado para México (Caballero y Cortés, 2001).

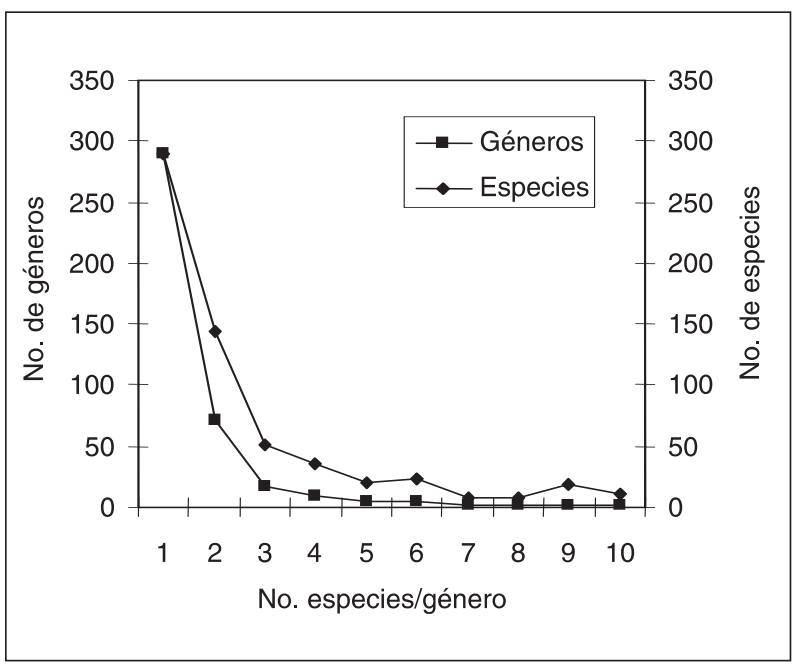

Figura 3. Distribución de frecuencias de los géneros y las especies con respecto al número de especies registradas por género. 
Dominancia CUltural De LaS Plantas Útiles de MoRelos

Cuadro 8. Distribución de las especies dominantes culturalmente por su origen y grado de manejo.

\begin{tabular}{|c|c|c|c|c|}
\hline \multirow[t]{3}{*}{ Grado de manejo } & \multicolumn{4}{|c|}{ Origen } \\
\hline & \multicolumn{2}{|c|}{ Introducidas } & \multicolumn{2}{|c|}{ Nativas } \\
\hline & Número & $\%$ & Número & $\%$ \\
\hline Cultivadas & 39 & 37.50 & 7 & 6.73 \\
\hline Cultivadas-naturalizadas & 3 & 2.88 & - & - \\
\hline Naturalizadas & 5 & 4.81 & - & - \\
\hline Cultivadas-zonas perturbadas & - & - & 5 & 4.81 \\
\hline Cultivadas-zonas perturbadas-silvestres & - & - & 2 & 1.92 \\
\hline Zonas perturbadas & - & - & 16 & 15.38 \\
\hline Silvestres & - & - & 8 & 7.69 \\
\hline Silvestres-cultivadas & - & - & 11 & 10.58 \\
\hline Silvestres-zonas perturbadas & - & - & 8 & 7.69 \\
\hline Total & $47^{*}$ & 45.19 & 57 & 54.81 \\
\hline
\end{tabular}

* incluye ocho especies que son originarias de las Antillas, Cuba o Sudamérica.

Cuadro 9. Dominancia cultural de los tipos de uso con base en su número de especies.

\begin{tabular}{|c|c|c|c|}
\hline \multirow[t]{2}{*}{ Tipo de uso } & \multirow[t]{2}{*}{ Clave } & \multicolumn{2}{|c|}{ Dominancia cultural } \\
\hline & & $\begin{array}{c}\text { Absoluta } \\
\text { (No. de especies) }\end{array}$ & $\begin{array}{c}\text { Relativa } \\
(\%)\end{array}$ \\
\hline Medicinal & M & 293 & 48.19 \\
\hline Ornamental & $\mathrm{O}$ & 255 & 41.94 \\
\hline Comestible & $\mathrm{C}$ & 169 & 27.80 \\
\hline Combustible & $\mathrm{CM}$ & 67 & 11.02 \\
\hline Construcción & $\mathrm{CN}$ & 53 & 8.72 \\
\hline Enseres domésticos & ED & 39 & 6.41 \\
\hline Cerco & CE & 38 & 6.25 \\
\hline Forraje & $\mathrm{F}$ & 30 & 4.93 \\
\hline Mágico-religioso & $M R$ & 29 & 4.77 \\
\hline Sombra & $\mathrm{S}$ & 28 & 4.61 \\
\hline Instrumento de trabajo & IT & 19 & 3.13 \\
\hline Instrumentos de labranza & IL & 17 & 2.80 \\
\hline Uso personal & UP & 13 & 2.14 \\
\hline Juguetes & $J$ & 12 & 1.97 \\
\hline Veterinario & V & 7 & 1.15 \\
\hline Artesanal & A & 6 & 0.99 \\
\hline Fruticultura & FR & 5 & 0.82 \\
\hline Instrumentos musicales & $\mathrm{IM}$ & 5 & 0.82 \\
\hline Tintoreo & $\tilde{N}$ & 4 & 0.66 \\
\hline Antorcha & $\mathrm{CH}$ & 2 & 0.33 \\
\hline Repelente de insectos & $\mathrm{R}$ & 2 & 0.33 \\
\hline Aromatizante & Z & 2 & 0.33 \\
\hline Cultivar hongos & $\mathrm{H}$ & 1 & 0.16 \\
\hline Papel & $\mathrm{L}$ & 1 & 0.16 \\
\hline Lavar ropa & LV & 1 & 0.16 \\
\hline Sellar cubetas & LL & 1 & 0.16 \\
\hline Abono & $\mathrm{N}$ & 1 & 0.16 \\
\hline
\end{tabular}

Dominancia cultural de las familias botánicas en los diferentes tipos de uso. - En el grupo de especies con uso medicinal (apéndice 1) sobresale la familia Asteraceae con $11.3 \%$ de dominancia relativa (33 especies de dominancia absoluta), Fabaceae con 6.83 (20\%) y Lamiaceae con 5.80 (17\%). Por lo que respecta a las plantas comestibles destaca la familia Fabaceae con una dominancia absoluta de 21 especies (12.4\%), así como Rosaceae con 12 (4.7\%) y Rutaceae con 10 (5.9\%), mientras que en el uso ornamental dominan Fabaceae con 19 especies (7.5\%), Arecaceae con $12(4.7 \%)$ y Moraceae con $10(3.9 \%)$. Cabe señalar que la familia Fabaceae ocupa los primeros lugares en 10 de los 12 tipos de uso que poseen más del $30 \%$ de su dominancia en familias con una o dos especies.

La distribución de la dominancia relativa de las familias que contribuyen con una y dos especies se comporta conforme al siguiente gradiente: los usos medicinal y ornamental tienen menos de $30 \%$ de su dominancia relativa distribuida en estas familias. A continuación están los usos comestible y para la construcción con menos de 40\%. En seguida se encuentran con los usos mágico-religioso, combustible, para la elaboración de enseres domésticos, forraje y cerco con menos de $50 \%$. Dicho gradiente continúa con el uso destinado a producir sombra $(<70 \%)$, para elaborar instrumentos de trabajo o de labranza $(<100 \%)$ y los 15 tipos de uso restantes (100\%).

\section{Discusión}

Método. Los índices usados en ecología de comunidades se adecuan con relativa facilidad para ser utilizados en la evaluación cuantitativa de información etnobotánica, como se ha demostrado por otros autores (Rico-Gray, 1991; Figueiredo et al., 1993; Begossi, 1996; Figueiredo et al., 1997; Benz et al., 2000; Begossi et al., 2002) y en la pre- 
sente colaboración. Esta evaluación requiere de un amplio marco conceptual (Caballero, 1993) que nos permita explicar de manera objetiva los valores obtenidos; se deben incluir aspectos relacionados con la heterogeneidad ambiental donde se realizaron los trabajos, la diferencia de enfoques y el tipo de grupo social con el que se hicieron las entrevistas (diferencias de edad, sexo, actividad ecónomica, etc.), además de los aspectos históricos y de dinámica social.

Riqueza de las plantas útiles en el estado de Morelos. El número de plantas con uso calculadas para México es de 7,000 (Caballero y Cortés, 2001), lo que representa 23.3\% del total calculado para el país. El porcentaje obtenido para Morelos sugiere que todavía existen alrededor de 200 especies cuyo uso no se ha descrito. Este número sería mayor si consideramos que el listado a partir del cual se ha elaborado el presente análisis surgió de fuentes de lo más diversas en intereses, algunas de ellas enfocadas al estudio de huertos familiares, otras más con énfasis en ecología o sistemática y el resto dirigidas a la búsqueda de recursos empleados para satisfacer necesidades específicas como la alimentación (ver cuadro 1).

A pesar de su pequeña extensión territorial, la riqueza relativa de especies útiles registrada para Morelos es mayor que la señalada para otras regiones que difieren cultural y ambientalmente. Sin embargo, se debe tener en cuenta que $45.2 \%$ de las especies que dominan culturalmente (cuadro 8) son introducidas. En este sentido se justifica la necesidad de continuar con la investigación etnobotánica, especialmente si consideramos la velocidad en los cambios de uso del suelo de forestal o agrícola a urbano en Morelos. Dichos cambios promovidos por la subvaluación económica de los bienes y servicios forestales (United Nations Environmental Programme, 2001), nos obligan a mostrar a la sociedad la enorme variedad de recursos vegetales con uso (productos forestales no maderables) cuyo aprovechamiento sustentable tiene el enorme potencial de contribuir en el desarrollo sustentable (Thadani, 2001, Shackleton et al., 2002; El-Hilaly et al., 2003) como una alternativa ante la venta indiscriminada de tierras y su consecuente urbanización.

Dominancia cultural de las plantas útiles en el estado de Morelos. Asteraceae y Fabaceae son las dos familias dominantes por su número de especies útiles en Morelos. Además, son las más numerosas en México y poseen una importante proporción de elementos endémicos (Rzedowski, 1992b). Asteraceae es la familia más numerosa en el mundo no sólo por su riqueza específica y genérica, sino también por su abundancia (Rzedowski, 1992a). Asimismo, debido a su diversidad y endemismo en las diferentes regiones ecológicas, incluyendo entre otras al Eje Volcánico Transversal, México es considerado como un centro de diversificación de dicha familia (Turner y Nesom, 1998). Por lo que respecta a Fabaceae, se trata de la segunda familia más grande de México debido fundamentalmente a la explosiva especiación de algunos de sus géneros. Además, México posee algunos centros de endemismo como la cuenca del río Balsas, y por ello está considerado como una región de diferenciación secundario de este grupo (Sousa y Delgado, 1998).

Ambas familias ofrecen un amplio espectro de compuestos con uso potencial, mismos que les otorgan una gran adaptabilidad a ecosistemas perturbados, así como un gran potencial económico y genético (Martínez, 1991). En este sentido, cabe destacar que Morelos queda comprendido en el área de distribución de los centros de diversificación y endemismo de ambas familias, lo cual indudablemente debe contribuir en la riqueza de los recursos vegetales con uso.

En el tercer sitio de dominancia está la familia Lamiaceae, un grupo reconocido por su porcentaje de endemismos específicos en México (>77\%) y su contenido de terpenos empleados con fines alimenticios y medicinales (Ramamoorthy y Elliot, 1998). Destacan además otras cuatro familias, dos de ellas sobresalientes por poseer especies valiosas por su uso ornamental (Moraceae y Arecaceae) y dos más por ser fuente de frutales que se comercializan ampliamente (Rutaceae y Rosaceae).

Por otra parte, seis de las 10 familias dominantes por su número de especies útiles se encuentran en la lista de las nueve familias más numerosas de la flora de Morelos, incluyendo Fabaceae, Asteraceae, Lamiaceae, Solanaceae, Euphorbiaceae y Poaceae. Sin embargo, a pesar de la preponderancia de estas familias, su contribución es semejante a la obtenida del conjunto de familias con pocas especies útiles. Asimismo, existe un grupo de familias (Cactaceae, Arecaceae, Rosaceae y Rutaceae) dominantes por su número de especies útiles que no aparecen entre las más numerosas de la flora de Morelos.

Al comparar la lista de las 12 familias dominantes que contienen la mayoría de las 3,500 especies útiles que se han registrado para México (Caballero y Cortés, 2001) con el listado de especies útiles de Morelos, se observa que hay nueve familias dominantes comunes: Fabaceae, Asteraceae, Lamiaceae, Solanaceae, Euphorbiaceae, Poaceae, Cactaceae, Rosaceae y Rutaceae. Por lo tanto, el patrón de dominancia encontrado para Morelos coincide con el nacional. Esto podría indicar que existe un conjunto de recursos de uso generalizado, debido probablemente al intercambio de conocimiento o a la semejanza en los patrones de experimentación aplicados a los recursos con el fin de obtener satisfactores (Goodman y Hobbs, 1988).

Sin embargo, la presencia de un grupo de familias comunes no implica descartar la relevancia de otras que resultan de utilidad, dadas las condiciones ambientales y culturales particulares que caracterizan al estado de 
Morelos. Aunado a lo anterior, es importante mencionar que salvo la familia Fabaceae, las restantes no conservan su preponderancia en todas las categorías de uso. Por ejemplo, en el caso del uso ornamental el segundo lugar en dominancia es ocupado por Arecaceae, mientras que las familias Rosaceae y Rutaceae son segundas y terceras en el uso comestible. En este sentido, se coincide con la propuesta que los recursos vegetales con uso han resultado de la combinación de un proceso selectivo basado en la cultura y de la disponibilidad (riqueza y abundancia) de ciertas familias (Girach et al., 1999; Moerman y Estabrook, 2003).

Este hallazgo tiene implicaciones en términos de desarrollo sustentable, en tanto las propuestas para lograrlo deberían considerar la relevancia y la particularidad de las condiciones ambientales y culturales de las comunidades y pueblos (Anónimo, 1998; Casas et al., 2001), ya que con frecuencia se ignoran aún sus derechos y antecedentes históricos (Hernández-X., 1993). Por lo tanto, se debería garantizar el fomento y el rescate de los procesos selectivos de los recursos, así como de su sustento cultural y ambiental, ya que como se ha mostrado en numerosas ocasiones, la interacción de la sociedad y el ambiente ha resultado en la generación de múltiples satisfactores (Martínez-Alfaro et al., 1995; Caballero y Cortés, 2001; Casas et al., 2001), en la ordenación del ambiente y el desarrollo sustentable (United Nations, 1992).

Los géneros dominantes se pueden diferenciar en tres grupos, uno conformado por especies introducidas, el segundo con especies nativas y el tercero cuya diversificación se promueve particularmente en ambientes perturbados socialmente. En el primer grupo se tiene a Citrus, cuyas especies son nativas del sureste de Asia y de Malasia; la mayoría de éstas son ampliamente cultivadas con fines frutícolas u ornamentales, y algunas están naturalizadas (Porter y Elias, 1979). En el segundo se incluyen Bursera, Acacia, Euphorbia, Ipomoea, Salvia y Solanum, géneros caracterizados por su riqueza de especies o por su endemismo (McDonald, 1991; Rzedowski, 1992ab). Los cuatro primeros están muy diversificados en la tierra caliente de la vertiente pacífica (Rzedowski, 1992ab) y el quinto en las zonas montañosas de México (Ramamoorthy y Elliott, 1998). En el tercero se cuenta con Euphorbia, género que incluye malezas nativas en proceso de evolución (Rzedowski, 1992a).

Dominancia cultural de las plantas útiles en el estado de Morelos. Los habitantes de Morelos que forman parte de nuestro universo de estudio satisfacen sus necesidades en proporciones semejantes con especies introducidas (45.2\%) y nativas $(54.8 \%)$. Estas especies se distribuyen a lo largo de un gradiente de manejo que va desde el cultivo $(64.4 \%)$, pasando por las especies que se ven favorecidas en ambientes perturbados socialmente, como las parcelas en descanso (29.8\%), y las silvestres, que se desarrollan en ambientes relativamente conservados $(27.9 \%)$.

La contribución de las especies introducidas, que es similar a la registrada en el norte de Sudamérica (Bennett y Prance, 2000), probablemente se relaciona entre otras cosas con el grado de transculturación de los habitantes de las comunidades y las regiones estudiadas, ya que $45.8 \%$ (11) de los trabajos consultados para este trabajo se realizaron en la zona central de Morelos (ver cuadro 1), donde históricamente el proceso de urbanización se ha presentado con una mayor intensidad, acompañado con la pérdida de recursos naturales y la transformación del conocimiento tradicional asociado a éste. Es decir, es en esta zona donde se han adoptado más plantas útiles que forman aportaciones de otras culturas, fundamentalmente la asiática (apéndice 2). El cambio cultural es una forma respuesta social ante un ambiente que se transforma continuamente (Caballero, 1993). En este sentido, sería interesante tratar de evaluar hasta dónde este cambio ha servido para enriquecer la cultura morelense o para favorecer su erosión.

Es importante destacar la obtención de satisfactores a partir de ambientes sujetos a diferentes grados de manejo. Además de los recursos producto del trabajo en ambientes creados socialmente como los huertos caseros, destaca la contribución de los ambientes perturbados (por ejemplo, el borde de los caminos o parcelas) y los bosques secundario. Todos éstos son espacios donde se promueve la presencia de plantas usadas como fuente de satisfactores (Balée, 1989; Casas, 2001), de manera semejante a lo que se ha encontrado en dos comunidades zapotecas y mixes de Oaxaca (Frei et al., 2000), en Haití (Weniger et al., 1986) y en Bolivia (Dewalt et al., 1999).

Así se pone de manifiesto el potencial de los diferentes ambientes que integran el paisaje, incluyendo aquellos que podrían ser considerados como perdidos por el nivel de perturbación en el que se encuentran. Este potencial tiene sustento en la cultura de las comunidades, misma que incluye no sólo el conocimiento sobre el uso y el manejo de los recursos, sino las formas de organización social que garantizan la generación, la transmisión y el resguardo del mismo (Leff, 1994).

Dominancia cultural de los diferentes tipos de uso. Con base en el número de tipos de uso es posible afirmar que en el estado se cuenta con una amplia gama de productos que satisfacen las más variadas necesidades de la sociedad morelense.

Los usos principales en Morelos coinciden con los señalados a nivel nacional: el comestible y el medicinal (Caballero y Cortés, 2001), ambos esenciales para la supervivencia y reproducción social. De igual forma, es importante resaltar la estrecha relación que existe entre ambos tipos de uso, facilitando el libre flujo de especies en un doble sentido; es decir, que una especie medicinal se pruebe como alimenticia o viceversa. Lo anterior se expli- 


\section{Columba Monroy-Ortiz y Rafael Monroy}

ca argumentando la falta de una clara división tradicional entre ambos tipos de uso (Moerman, 1996; Bennett y Prance, 2000; Pieroni, 2000), así como en la semejanza de las propiedades usadas con fines selectivos (color, sabor y apariencia) (Leonti et al., 2002).

El tercer tipo de uso dominante en Morelos es el ornamental, cuya preponderancia probablemente esté vinculada a las condiciones climáticas favorables para el cultivo de plantas que hay en el estado y a la importancia económica de esta actividad. Con respecto a este uso, es importante mencionar que se ha documentado la transición en el empleo de una especie con fines ornamentales a medicinales; es decir, algunas veces las especies introducidas exclusivamente como ornamentales son sometidas a evaluaciones empíricas para conocer su potencial medicinal en el contexto de un grupo cultural diferente (Bennett y Prance, 2000). La existencia de esta transición nos demuestra la importancia de no subestimar los tipos de uso considerados como no dominantes y prescindibles. Tal vez en este mismo sentido es necesario analizar si este tipo de flujo se da con otros usos, porque de ser así, al perder un recurso vegetal, el conocimiento tradicional asociado a éste, o a ambos, se tendría un efecto multiplicador que incrementaría considerablemente las tasas de erosión cultural.

Por otra parte, conforme disminuye la dominancia de un uso es mayor la presencia de familias con una o dos especies. Probablemente se trata de usos demandantes de características tan específicas que pocas especies las pueden satisfacer, o bien, son usos que al resultar menos prioritarios han requerido menos experimentación, lo que ha ocasionado el descubrimiento de menos recursos que para otros tipos de uso. Una opción más podría ser que se trate de usos de relevancia local, para los cuales resultan de mayor importancia los recursos de distribución restringida.

\section{Agradecimientos}

A Jorge A. Meave y a los dos revisores anónimos, quienes enriquecieron este trabajo con sus valiosas sugerencias. A Rafael Monroy Ortiz por su apoyo en la elaboración de la figura 1 .

\section{Literatura citada}

Agelet A. y Valles J. 2001. Studies on pharmaceutical ethnobotany in the region of Pallars (Pyrenees, Catalonia, Iberian Peninsula). Part. I. General results and new or very rare medicinal plants. Journal of Ethnopharmacology 77:57-70.

Aguilar M.L. 1993. Los agroecosistemas frutícolas tradicionales de Itzamatitlán, municipio de Yautepec, Morelos. Tesis de Licenciatura, Facultad de Ciencias Biológicas, Universidad Autónoma del Estado de Morelos, Cuernavaca, Mor., 69 pp.

Aguirre E. 1993. Plantas útiles para la elaboración de artesanías en el estado de Morelos. Tesis de Licenciatura, Facultad de Ciencias Biológicas, Universidad Autónoma del Estado de
Morelos, Cuernavaca, Mor., 68 pp.

Ali-Shtayeh M.S., Yaniv Z. y Mahajna J. 2000. Ethnobotanical survey in the Palestinian area: a classification of the healing potential of medicinal plants. Journal of Ethnopharmacology 73:221-232.

Altamirano D. 2001. Manual de Plantas Urbanas, Centro Histórico de Cuernavaca. Facultad de Arquitectura, Universidad Autónoma del Estado de Morelos, Cuernavaca, Mor.

Anónimo. 1998. Acuerdos sobre Derechos y Cultura Indígena. Mesa 1 de los Diálogos de San Andrés Sacamch'en. Frente Zapatista de Liberación Nacional, México, D.F.

Antonio E. 1997. Las asociaciones de frutales como resultado del manejo tradicional en los huertos de Xoxocotla. Tesis de Licenciatura, Facultad de Ciencias Biológicas, Universidad Autónoma del Estado de Morelos, Cuernavaca, Mor., 74 pp.

Ayala I. 1998. Etnobotánica, fuente de evidencias de la resistencia cultural en Tejalpa. Tesis de Licenciatura, Facultad de Ciencias Biológicas, Universidad Autónoma del Estado de Morelos, Cuernavaca, Mor., 61 pp.

Bajonero N. 1982. Contribución al conocimiento de la flora medicinal en la colonia Adolfo López Mateos, municipio de Tepalcingo, Morelos. Tesis de Licenciatura, Escuela de Ciencias Biológicas, Universidad Autónoma del Estado de Morelos, Cuernavaca, Mor., 47 pp.

Balée W. 1989. The culture of Amazonian forests. Advances in Economic Botany 7:1-21.

Barrau J. 1981. Plants and man on the threshold of the twenty-first century. XII International Botanical Congress, Closing Ceremony, Sidney, Australia.

Begossi A. 1996. Use of ecological methods in ethnobotany: diversity indices. Economic Botany 50:280-289.

Begossi A., Hanazaki N. y Tamashiro J. 2002. Medicinal plants in the Atlantic forest (Brazil): knowledge, use, and conservation. Human Ecology 30:281-299.

Bello J. y Vilchis M. 1989. Alternativas de manejo ecológico de las especies maderables en San Juan Tlacotenco, municipio de Tepoztlán, Morelos. Tesis de Licenciatura, Facultad de Ciencias Biológicas, Universidad Autónoma del Estado de Morelos, Cuernavaca, Mor., 80 pp.

Bennett B.C. y Prance G. 2000. Introduced plants in the indigenous pharmacopoeia of northern South America. Economic Botany 54:90-102.

Benz B.F., Cevallos J., Santana F., Rosales J. y Graf S. 2000. Losing knowledge about plant use in the Sierra de Manantlán Biosphere Reserve, Mexico. Economic Botany 54:183-191.

Bonilla-Barbosa J.R. y Villaseñor J.L. 2003. Catálogo de la Flora del Estado de Morelos. Centro de Investigaciones Biológicas, Universidad Autónoma del Estado de Morelos, Cuernavaca, Mor.

Brummitt R.K. y Powell C.E. 1992. Authors of Plant Names. Royal Botanic Gardens, Kew, Reino Unido.

Caballero J. 1993. El caso del uso y manejo de la palma de guano (Sabal spp.) entre los mayas de Yucatán. En: Leff E. y Carabias J. Eds. Cultura y Manejo Sustentable de los Recursos Naturales, pp. 203-248, Centro de Investigaciones Interdisciplinarias en Humanidades, Universidad Nacional Autónoma de México y Miguel Angel Porrúa, México, D.F.

Caballero J. y Cortés L. 2001. Percepción, uso y manejo tradicional de los recursos vegetales en México. En: Rendón B., 
Rebollar S., Caballero J. y Martínez M.A. Eds. Plantas, Cultura y Sociedad. Estudio sobre la Relación entre Seres Humanos y Plantas en los Albores del Siglo XXI, pp. 79-100, Universidad Autónoma Metropolitana, Unidad Iztapalapa y Secretaría del Medio Ambiente, Recursos Naturales y Pesca, México, D.F.

Casas A. 2001. Silvicultura y domesticación de plantas en Mesoamérica. En: Rendón B., Rebollar S., Caballero J. y Martínez M.A. Eds. Plantas, Cultura y Sociedad. Estudio sobre la Relación entre Seres Humanos y Plantas en los Albores del Siglo XXI, pp. 123-158, Universidad Autónoma Metropolitana, Unidad Iztapalapa y Secretaría del Medio Ambiente, Recursos Naturales y Pesca, México, D.F.

Casas A., Valiente-Banuet A., Viveros J.L., Caballero J., Cortés L., Dávila P., Lira R. y Rodríguez I. 2001. Plant resources of the Tehuacán-Cuicatlán Valley, Mexico. Economic Botany 55:129-166.

Cervantes L. y Valdés J. 1990. Plantas medicinales del distrito de Ocotlán, Oaxaca. Anales del Insituto de Biología, Universidad Nacional Autónoma de México, Serie Botánica 60:85-103.

Colín H. 1990. Estudio de la relación de los agroecosistemas frutícolas con la calidad de vida humana en Tetela del Volcán, Morelos, México. Tesis de Licenciatura, Facultad de Ciencias Biológicas, Universidad Autónoma del Estado de Morelos, Cuernavaca, Mor., 82 pp.

Convenio sobre Diversidad Biológica. 1992. Secretaría del Convenio sobre Diversidad Biológica. Programa de las Naciones Unidas para el Medio Ambiente. http://www.biodiv.org/doc/legal/cbd-es.pdf

Cronquist A. 1981. An Integrated System of Classification of Flowering Plants. Columbia University Press, Nueva York.

Chávez C. 1979. Estudio preliminar de etnobotánica y algunas implicaciones ecológicas en los ejidos: El Higuerón, Jicarero, Panchimalco y Tlatenchi de Jojutla, Morelos, México. Tesis de Licenciatura, Escuela de Ciencias Biológicas, Universidad Autónoma del Estado de Morelos, Cuernavaca, Mor., 35 pp.

Dahlgren R.M.T., Cliffoird H.T. y Yeo P.F. 1985. The Families of the Monocotyledons. Springer-Verlag, Nueva York.

Davidse G., Sousa M. y Chater A.O. Eds. 1995. Flora Mesoamericana. Psilotaceae a Salviniaceae. Universidad Nacional Autónoma de México-Missouri Botanical GardenThe Natural History Museum, México, D.F.

del Amo S. y Anaya A.L. 1982. Importancia de la sistematización de la información sobre plantas medicinales. Biotica 7:293304.

Dewalt S.J., Bourdy G., Chávez R. y Quenevo C. 1999. Ethnobotany of the Tacana: quantitative inventories of two permanent plots of northwestern Bolivia. Economic Botany 53:237-260.

El-Hilaly J., Hmammouchi M. y Lyoussi B. 2003. Ethnobotanical studies and economic evaluation of medicinal plants in Taounate province (Northern Morocco). Journal of Ethnopharmacology 86:149-158.

Espinosa J. 2001. Gymnospermae. En: Calderón de Rzedowski G. y Rzedowski J. Eds. y autores principales. Flora Fanerogámica del Valle de México, pp. 44-55, Instituto de Ecología, A.C., Centro Regional del Bajío-Comisión Nacional para el Conocimiento y Uso de la Biodiversidad, Pátzcuaro, Mich.

Figueiredo G., Leitão-Filho F. y Begossi A. 1993. Ethnobotany of atlantic forest coastal communities: diversity of plant uses in
Gamboa (Itacuruça Island, Brazil). Human Ecology 21:419430.

Figueiredo G., Leitão-Filho F. y Begossi A. 1997. Ethnobotany of atlantic forest coastal communities. II. Diversity of plants uses at Sepetiba bay (SE Brasil). Human Ecology 25:353-360.

Flores A. 1988. Los árboles ornamentales de la ciudad de Cuernavaca, Morelos. Tesis de Licenciatura, Facultad de Ciencias Biológicas, Universidad Autónoma del Estado de Morelos, Cuernavaca, Mor., 205 pp.

Frei B., Sticher O. y Heinrich M. 2000. Zapotec and mixe use of tropical habitats for securing medicinal plants in Mexico. Economic Botany 54:73-81.

Girach R.D., Shaik A.A., Sing S.S. y Ahmad M. 1999. The medicinal flora of Similipahar forests, Orissa state, India. Journal of Ethnopharmacology 65:165-172.

Gispert M. 1992. La etnobotánica en Latinoamérica. En: Resúmenes de Participación. Etnobotánica 92, p. 17, Córdoba, España.

Goodman S. y Hobbs J.J. 1988. The ethnobotany of the egyptian eastern desert: a comparison of common plant usage between two culturally distinct bedouin groups. Journal of Ethnopharmacology 23:73-89.

Hernández E. 1983. Contribución al conocimiento de la relación planta-hombre en el ejido de Tecajec, municipio de Yecapixtla, Morelos. Tesis de Licenciatura, Escuela de Ciencias Biológicas, Universidad Autónoma del Estado de Morelos, Cuernavaca, Mor., 109 pp.

Hernández-X. E. 1985. Exploración etnobotánica y su metodología. Xolocotzia. Revista de Geografía Agrícola Tomo I:189-194.

Hernández-X. E. 1993. Utilización de los recursos vegetales en México. En: Guevara S., Moreno-Casasola P. y Rzedowski J. Eds. Logros y Perspectivas del Conocimiento de los Recursos Vegetales de México en Vísperas del Siglo XXI, pp. 57-62, Instituto de Ecología, Xalapa, Veracruz.

Heywood V. 1992. La etnobotánica y la estrategia mundial para la conservación. En: Resúmenes de Participación. Etnobotánica 92, pp. 15-16, Córdoba, España.

Krebs C. 1985. Ecología. Estudio de la Distribución y Abundancia. Editorial Harla, México, D.F.

Leff E. 1994. La dimensión cultural del manejo integrado, sustentable y sostenido de los recursos naturales. En: Leff E. y Carabias J. Eds. Cultura y Manejo Sustentable de los Recursos Naturales, pp. 55-88, Centro de Investigaciones Interdisciplinarias en Humanidades, Universidad Nacional Autónoma de México y Miguel Angel Porrúa, México, D.F.

Leonti M., Sticher O. y Heinrich M. 2002. Medicinal plants of the popoluca, Mexico: organoleptic properties as indigenous selection criteria. Journal of Ethnopharmacology 81:307-315.

Ley General del Equilibrio Ecológico y la Protección al Ambiente (última reforma 13/06/2003). 2003. http://www.cddhcu.gob.mx/leyinfo/pdf/148.pdf.

Li R.W., Myers S.P., Leach D.N., Lin D. y Leach G. 2003. A cross-cultural study: anti-inflammatory activity of Australian and Chinese plants. Journal of Ethnopharmacology 85:25-32.

McDonald A. 1991. Origin and diversity of Mexican Convolvulaceae. Anales del Instituto de Biología, Universidad Nacional Autónoma de México, Serie Botánica 62:65-82.

Maldonado B. y Monroy R. 1989a. Edible plants of tropical deciduos forest in State of Morelos, Mexico. Journal of 
Ethnobiology 9:245.

Maldonado B. y Monroy R. 1989b. La selva baja caducifolia en el estado de Morelos. Ciencia y Desarrollo XV:41-49.

Maldonado D. 1990. Cuauhnáhuac y Huaxtepec (Tlahuicas y Xochimilcas en el Morelos Prehispánico). Centro Regional de Investigaciones Multidisciplinarias, Universidad Nacional Autónoma de México, México, D.F.

Martínez D. 1985. Las cactáceas del estado de Morelos. Tesis de Licenciatura, Escuela de Ciencias Biológicas, Universidad Autónoma del Estado de Morelos, Cuernavaca, Mor., 176 pp.

Martínez M.A. 1991. Cinco familias de plantas con potencial económico y genético para México. En: Ortega R., Palomino G., Castillo F., González V. y Livera M. Eds. Avances en el Estudio de los Recursos Fitogenéticos de México, pp. 53-62, Sociedad Mexicana de Fitogenética (SOMEFI), A.C., Chapingo, Estado de México.

Martínez-Alfaro M.A., Evangelista V., Mendoza M., García G.M., Toledo G. y Wong A. 1995. Catálogo de Plantas Útiles de la Sierra Norte de Puebla, México. Instituto de Biología, Universidad Nacional Autónoma de México, México, D.F.

Martínez-Lirola M.J., González-Tejero M.R. y Molero-Mesa J. 1996. Ethnobotanical resources in the province of Almería, Spain: Campos de Nijar. Economic Botany 50:40-56.

Matosic R.L. 1991. Conocimiento y uso tradicional de la flora de Nepopualco, municipio de Totolapan, Morelos. Tesis de Licenciatura, Escuela de Ciencias Biológicas, Universidad Autónoma del Estado de Morelos, Cuernavaca, Mor., 69 pp.

Missouri Botanical Garden. 2002. Nomenclatural data base. VAST (vascular tropicos). Release 1.5. http://mobot.mobot.org/W3T/Search/vast.html.

Moerman D.E. 1996. An analysis of the food plants and drug plants of native North America. Journal of Ethnopharmacology 52:1-22.

Moerman D.E., Pemberton R.W., Kiefer D. y Berlin B. 1999. A comparative analysis of five medicinal floras. Journal of Ethnobiology 19:49-67.

Moerman D.E. y Estabrook G.F. 2003. Native Americans choice of species for medicinal use is dependent on plant family: confirmation with meta-significance analysis. Journal of Ethnopharmacology 87:51-59.

Monroy R., Palacios G. y González E. 1986. Comercialización de plantas, evidencia de su diversidad. Expresión Universitaria 12:16-20.

Monroy R., Taboada M., Maldonado B., Oliver R. y Colín O. 1992. Características del medio físico biótico. En: Oswald U. Ed. Mitos y Realidades del Morelos Actual, pp. 37-64, Centro Regional de Investigaciones Multidisciplinarias, Universidad Nacional Autónoma de México, Cuernavaca, Mor.

Monroy-Ortiz C. 1997. La leña como recurso energético. Implicaciones ecológicas y etnobotánicas. Tesis de Maestría, Facultad de Ciencias, Universidad Nacional Autónoma de México, México, D.F., 114 pp.

Monroy-Ortiz C. y Monroy R. 2001. Dominancia de los valores de uso asignados a los recursos vegetales utilizados en el estado de Morelos. En: Libro de Resúmenes, IV Congreso Mexicano de Etnobiología, 30 octubre-2 noviembre de 2001, p. 32, Huejutla, Hgo.

Muthcnick P.A. y McCarthy B.C. 1997. An ethnobotanical analysis of the tree species common to the subtropical moist forests of the Petén, Guatemala. Economic Botany 51:158-183.
Pérez V.M. 1982. Etnobotánica de la región Huautla, municipio de Tlaquiltenango, Morelos. Tesis de Licenciatura, Escuela de Ciencias Biológicas, Universidad Autónoma del Estado de Morelos, Cuernavaca, Mor., 49 pp.

Phillips O. 1996. Some quantitative methods for analyzing ethnobotanical knowledge. En: Alexiades M. Ed. Selected Guidelines for Ethnobotanical Research: a Field Manual, pp. 171-197, The New York Botanical Garden, Nueva York.

Phillips O. y Gentry A.H. 1993. The useful plants of Tamboata, Peru: I. Statistical hypotheses tests with a new quantitative technique. Economic Botany 47:15-32.

Pieroni A. 2000. Medicinal plants and food medicines in the folk traditions of the upper Lucca Province, Italy. Journal of Ethnopharmacology 70:235-273.

Porter D.M. y Elias T.S. 1979. Rutaceae. Flora of Panama. Parte IX. Annals of the Missouri Botanical Garden 66:123-164.

Provencio E. y Carabias J. 1993. El enfoque del desarrollo sustentable. En: Azuela A., Carabias J., Provencio E. y Quadri G. Eds. Desarrollo Sustentable. Hacia una Política Ambiental, pp. 3-12, Universidad Nacional Autónoma de México, México, D.F.

Quezada M.R. 1990. Diseño y establecimiento del jardín botánico escolar de la escuela preparatoria "Valentín Gómez Farias" en el municipio de Emiliano Zapata, Morelos, México. Tesis de Licenciatura, Facultad de Ciencias Biológicas, Universidad Autónoma del Estado de Morelos, Cuernavaca, Mor., 78 pp.

Raja D., Blanché C. y Vallès J. 1997. Contribution to the knowledge of the pharmaceutical ethnobotany of La Segarra region (Catalonia, Iberian Peninsula). Journal of Ethnopharmacology 57:149-160.

Rico-Gray V., Chemas A. y Mandujano S. 1991. Uses of tropical deciduous forest species by the Yucatecan Maya. Agroforestry Systems 14:149-161.

Ramamoorthy T.P. y Elliott M. 1998. Lamiaceae de México: diversidad, distribución, endemismo y evolución. En: Ramamoorthy T.P., Bye R., Lot A. y Fa J. Eds. Diversidad Biológica de México. Orígenes y Distribución, pp. 501-526, Instituto de Biología, Universidad Nacional Autónoma de México, México, D.F.

Rzedowski J. 1978. Vegetación de México. Limusa, México, D.F.

Rzedowski J. 1992a. Diversidad y origen de la flora fanerogámica de México. Acta Zoologica Mexicana Vol. Esp.:313-335.

Rzedowski J. 1992b. El endemismo en la flora fanerogámica mexicana: una apreciación analítica preliminar. Acta Zoologica Mexicana Vol. Esp.:337-359.

Said O., Khalil K., Fulder S. y Azaizeh H. 2002. Ethnopharmacological survey of medicinal herbs in Israel, the Golan Heights and the West Bank region. Journal of Ethnopharmacology 83:251-265.

Salazar M.E. 1994. Elementos agroecológicos de los huertos tradicionales de Emiliano Zapata, Morelos. Tesis de Licenciatura, Facultad de Ciencias Biológicas, Universidad Autónoma del Estado de Morelos, Cuernavaca, Mor., 103 pp.

Sánchez M. 1982. Estudio preliminar etnobotánico sobre las plantas medicinales utilizadas en la comunidad de Tetelcingo, municipio de Cuautla, Morelos, México. Tesis de Licenciatura, Escuela de Ciencias Biológicas, Universidad Autónoma del Estado de Morelos, Cuernavaca, Mor., 40 pp.

Shackleton S.E., Shackleton S.M., Netshiluvhi T.R., Geach B.S., Ballance A. y Fairbanks D.H.K. 2002. Use patterns and value 


\section{Dominancia CUltural DE LAS Plantas ÚtILES DE MoRelos}

of savanna resources in three rural villages in South Africa. Economic Botany 56:130-146.

Sousa M. y Delgado A. 1998. Leguminosas mexicanas: fitogeografía, endemismo y orígenes. En: Ramamoorthy T.P., Bye R., Lot A. y Fa J. Eds. Diversidad Biológica de México. Orígenes y Distribución, pp. 449-500, Instituto de Biología, Universidad Nacional Autónoma de México, México, D.F.

Taboada M. 1981. Aportación al conocimiento frutícola con enfoque etnobotánico y ecológico para el estado de Morelos. Tesis de Licenciatura, Escuela de Ciencias Biológicas, Universidad Autónoma del Estado de Morelos, Cuernavaca, Mor., 65 pp.

Thadani R. 2001. Internacional non-timber forest product issues. Journal of Sustainable Forestry 13:5-23.

Toledo V.M. 1982. La etnobotánica hoy: reversión del conocimiento, lucha indígena y proyecto nacional. Biótica 7:141-150.

Toledo V.M. y Argueta A. 1994. Naturaleza, producción y cultura en una región indígena de México: las lecciones de Pátzcuaro. En: Leff E. y Carabias J. Eds. Cultura y Manejo Sustentable de Recursos Naturales No Renovables, pp. 413-441, Centro de Investigaciones Interdisciplinarias en Humanidades, Universidad Nacional Autónoma de México y Miguel Ángel Porrúa, México, D.F.

Turner B.L. y Nesom G.L. 1998. Biogeografía, diversidad y situación de peligro o amenaza de Asteraceae en México. En: Ramamoorthy T.P., Bye R., Lot A. y Fa J. Eds. Diversidad Biológica de México. Orígenes y Distribución, pp. 545-561, Instituto de Biología, Universidad Nacional Autónoma de México, México, D.F.

United Nations Environmental Programme. 2001. Diversidad biológica forestal. Informe del Grupo Técnico Especial de Expertos en Diversidad Biológica Forestal. Convenio Sobre la
Diversidad Biológica. UNEP/CBD/SBSTTA/7/6, Montreal. United Nations. 1992. Anexo I. Declaración de Río sobre el medio ambiente y el desarrollo. En: Informe de la Conferencia de las Naciones Unidas sobre el Medio Ambiente y el Desarrollo (Río de Janeiro, 3 a 14 de junio de 1992). A/CONF.151/26 (Vol. I).

http://www.un.org/documents/ga/conf151/spanish/aconf151261 annex 1 s.htm.

Vasisht K. y Kumar V. 2002. Trade and Production of Herbal Medicines and Natural Health Products. International Center for Science and High Technology, Ambasciata d'Italia Budapest, United Nations Industrial Development Organization, Trieste, Italia.

Villa A.N. 1993. Estudio preliminar de la propagación y análisis bromatológico del tempesquistle Bumelia laetevirens Hemsl. Tesis de Licenciatura, Facultad de Ciencias Biológicas, Universidad Autónoma del Estado de Morelos, Cuernavaca, Mor., 57 pp.

Villegas J. 1979. Estudio preliminar de etnobotánica y algunas implicaciones ecológicas en los ejidos: Tequesquitengo, Tehuixtla, Río Seco y Chisco del Valle de Jojutla, Morelos, México. Tesis de Licenciatura, Escuela de Ciencias Biológicas, Universidad Autónoma del Estado de Morelos, Cuernavaca, Mor., 35 pp.

Von Wobeser G. 1989. La Formación de la Hacienda en la Época Colonial. El Uso de la Tierra y el Agua. Instituto de Investigaciones Históricas, Universidad Nacional Autónoma de México, México, D.F.

Weniger B., Rouzier M., Dagulh R., Henry D., Henrys J.H. y Anton R. 1986. La medicine populaire dans le plateau central d'Haiti. 2. Inventaire ethnopharmacologique. Journal of Ethnopharmacology 17:13-30.

Fecha de recepción: 24 de noviembre de 2003

Versión corregida: 12 de abril de 2004

Aceptado: 19 de abril de 2004 


\section{Columba Monroy-Ortiz y Rafael Monroy}

Apéndice 1. Distribución de la dominancia cultural relativa en cada uno de los usos, conforme al número de especies registradas por familia. La dominancia cultural relativa puede referirse a la familia o al conjunto de familias que tienen el mismo número de especies. Ver cuadro 9 para el significado de las letras correspondientes a los tipos de uso.

\begin{tabular}{|c|c|c|c|c|c|c|c|c|c|c|c|c|c|c|c|c|c|c|c|}
\hline \multirow{2}{*}{$\begin{array}{l}\text { Tipo } \\
\text { de } \\
\text { uso }\end{array}$} & \multicolumn{19}{|c|}{ (No. especies/familia) } \\
\hline & 1 & 2 & 3 & 4 & 5 & 6 & 7 & 8 & 9 & 10 & 11 & 12 & 13 & 15 & 17 & 19 & 20 & 21 & 33 \\
\hline M & 12.29 & 15.02 & 12.29 & 9.56 & $\begin{array}{l}3.42 \\
B I G, \\
R \cup B\end{array}$ & $\begin{array}{c}4.1 \\
C O V \\
M A V\end{array}$ & $\begin{array}{l}2.39 \\
V E R\end{array}$ & $\begin{array}{l}2.73 \\
B O R\end{array}$ & $\begin{array}{l}6.14 \\
\text { ROS, } \\
\text { RUT }\end{array}$ & & $\begin{array}{l}3.75 \\
E U P\end{array}$ & & $\begin{array}{l}4.44 \\
S O L\end{array}$ & & $\begin{array}{l}5.80 \\
L A M\end{array}$ & & $\begin{array}{l}6.83 \\
F A B\end{array}$ & & $\begin{array}{c}11.26 \\
A S T\end{array}$ \\
\hline $\mathrm{O}$ & 14.90 & 10.20 & 10.59 & 10.98 & $\begin{array}{c}7.84 \\
\text { ANA, } \\
B U R, \\
\text { OLE, } \\
\text { VER }\end{array}$ & $\begin{array}{c}4.70 \\
A S T \\
E U P\end{array}$ & $\begin{array}{c}8.25 \\
A R A, \\
R O S, \\
S O L\end{array}$ & $\begin{array}{l}9.42 \\
M Y T, \\
R U T, \\
A P O\end{array}$ & $\begin{array}{l}7.06 \\
B I G \\
C A C\end{array}$ & $\begin{array}{c}3.92 \\
\text { MOR }\end{array}$ & & $\begin{array}{l}4.71 \\
A R E\end{array}$ & & & & $\begin{array}{l}7.45 \\
F A B\end{array}$ & & & \\
\hline C & 13.61 & 18.93 & $\begin{array}{l}1.78 \\
A P I\end{array}$ & $\begin{array}{c}7.11 \\
A R E \\
L A M, \\
S A O\end{array}$ & $\begin{array}{l}5.92 \\
\text { ANA, } \\
\text { MYT }\end{array}$ & $\begin{array}{c}14.2 \\
\text { ANN, } \\
\text { CUC, } \\
\text { CHE, } \\
B R A\end{array}$ & $\begin{array}{l}8.28 \\
A S T \\
S O L\end{array}$ & $\begin{array}{l}4.73 \\
C A C\end{array}$ & & $\begin{array}{l}5.92 \\
R U T\end{array}$ & & $\begin{array}{l}7.10 \\
R O S\end{array}$ & & & & & & $\begin{array}{c}12.43 \\
F A B\end{array}$ & \\
\hline $\mathrm{CN}$ & 28.3 & $\begin{array}{l}11.31 \\
\text { ANA. } \\
\text { CAC, } \\
\text { ULM }\end{array}$ & $\begin{array}{c}16.98 \\
A S T, \\
F A G, \\
M E I\end{array}$ & $\begin{array}{l}15.1 \\
P I N \\
P O A\end{array}$ & & & & & & & & & & $\begin{array}{c}28.30 \\
\text { FAB }\end{array}$ & & & & & \\
\hline MR & 34.48 & $\begin{array}{l}11.31 \\
\text { ANA, } \\
\text { CAC, } \\
\text { ULM }\end{array}$ & $\begin{array}{c}16.98 \\
A S T, \\
F A G, \\
M E I\end{array}$ & $\begin{array}{l}15.1 \\
P I N \\
P O A\end{array}$ & & & & & & & & & & $\begin{array}{c}28.30 \\
F A B\end{array}$ & & & & & \\
\hline CM & 28.36 & 17.91 & $\begin{array}{c}8.96 \\
M E I, \\
M O R\end{array}$ & $\begin{array}{c}11.94 \\
\text { FAG } \\
\text { PIN }\end{array}$ & $\begin{array}{l}7.46 \\
B \cup R\end{array}$ & & & & & & & & & & $\begin{array}{c}25.37 \\
F A B\end{array}$ & & & & \\
\hline ED & 25.64 & $\begin{array}{c}20.52 \\
A R E, \\
A S T, \\
B I G, \\
C U C\end{array}$ & $\begin{array}{l}7.69 \\
B \cup R\end{array}$ & $\begin{array}{c}20.52 \\
F A B \\
P I N\end{array}$ & $\begin{array}{c}25.64 \\
M E I, \\
P O A\end{array}$ & & & & & & & & & & & & & & \\
\hline $\mathrm{F}$ & 40.00 & $\begin{array}{c}6.67 \\
M O R\end{array}$ & $\begin{array}{c}10.00 \\
A S T\end{array}$ & & & $\begin{array}{c}20.00 \\
F A B\end{array}$ & $\begin{array}{c}23.33 \\
P O A\end{array}$ & & & & & & & & & & & & \\
\hline CE & 28.95 & $\begin{array}{c}15.78 \\
B I G \\
B O M, \\
C A C\end{array}$ & $\begin{array}{l}7.89 \\
\text { ANA }\end{array}$ & $\begin{array}{c}10.53 \\
\text { PIN }\end{array}$ & $\begin{array}{c}13.16 \\
B \cup R\end{array}$ & $\begin{array}{c}23.68 \\
F A B\end{array}$ & & & & & & & & & & & & & \\
\hline S & 32.14 & $\begin{array}{c}28.56 \\
E U P, \\
M O R, \\
M Y T, \\
S O L\end{array}$ & $\begin{array}{c}10.71 \\
\text { BIG }\end{array}$ & $\begin{array}{c}28.58 \\
\text { ANA, } \\
\text { FAB }\end{array}$ & & & & & & & & & & & & & & & \\
\hline IL & 64.71 & $\begin{array}{c}11.76 \\
\text { FAG }\end{array}$ & & $\begin{array}{l}23.53 \\
\text { PIN }\end{array}$ & & & & & & & & & & & & & & & \\
\hline IT & 63.15 & $\begin{array}{c}10.53 \\
B \cup R\end{array}$ & & & $\begin{array}{c}26.32 \\
\text { FAB }\end{array}$ & & & & & & & & & & & & & & \\
\hline
\end{tabular}

ANA, Anacardiaceae; ANN, Annonaceae; API, Apiaceae; APO, Apocynaceae; ARA, Araceae; ARE, Arecaceae; AST, Asteraceae; BIG, Bignoniaceae; BOM, Bombacaceae; BOR, Boraginaceae; BRA, Brassicaceae; BUR, Burseraceae; CAC, Cactaceae; CHE, Chenopodiaceae; COV, Convolvulaceae; CUC, Cucurbitaceae; EUP, Euphorbiaceae; FAB, Fabaceae; FAG, Fagaceae; LAM, Lamiaceae; MAV, Malvaceae; MEI, Meliaceae; MOR, Moraceae; MYT, Myrtaceae; OLE, Oleaceae; PIN, Pinaceae; POA, Poaceae; ROS, Rosaceae; RUB, Rubiaceae; RUT, Rutaceae; SAO, Sapotaceae; SOL, Solanaceae; ULM, Ulmaceae; VER, Verbenaceae. 
Apéndice 2. Dominancia cultural de las plantas útiles en el estado de Morelos.

\begin{tabular}{|c|c|c|c|c|c|c|}
\hline \multirow[t]{2}{*}{ Familia } & \multirow[t]{2}{*}{ Nombre científico } & \multicolumn{2}{|c|}{$\begin{array}{l}\text { Dominancia cultural } \\
\text { (Citas) }\end{array}$} & \multirow[t]{2}{*}{ Manejo } & \multirow[t]{2}{*}{ Origen } & \multirow[t]{2}{*}{$\begin{array}{l}\text { Región de origen } \\
\text { o distribución }\end{array}$} \\
\hline & & No. & $\%$ & & & \\
\hline Lauraceae & Persea americana Mill. & 13 & 50.00 & C & $\mathrm{N}$ & Caribe, Guatemala, México \\
\hline Myrtaceae & Psidium guajava L. & 13 & 50.00 & $\mathrm{C}$ & $\mathrm{N}$ & América tropical \\
\hline Fabaceae & Pithecellobium dulce (Roxb.) Benth. & 12 & 46.15 & $\mathrm{P}$ & $\mathrm{N}$ & México a Sudamérica \\
\hline Rutaceae & Citrus aurantifolia (Christm.) Swingle & 12 & 46.15 & $\mathrm{C}$ & I & Norte y este de India \\
\hline Asteraceae & Senecio salignus DC. & 11 & 42.31 & $\mathrm{P}$ & $\mathrm{N}$ & $\begin{array}{c}\text { Sur de Estados Unidos a } \\
\text { Centroamérica }\end{array}$ \\
\hline Bignoniaceae & Crescentia alata Kunth & 11 & 42.31 & $\mathrm{P}$ & $\mathrm{N}$ & América \\
\hline Chenopodiaceae & Chenopodium ambrosioides L. & 11 & 42.31 & $\mathrm{CP}$ & $\mathrm{N}$ & América tropical \\
\hline Rutaceae & Ruta chalepensis L. & 11 & 42.31 & $\mathrm{C}$ & I & Mediterráneo \\
\hline Anacardiaceae & Mangifera indica L. & 10 & 38.46 & $\mathrm{C}$ & I & Sudeste de Asia \\
\hline Caricaceae & Carica papaya L. & 10 & 38.46 & SC & $\mathrm{N}$ & Sur de México a Costa Rica \\
\hline Fabaceae & Erythrina americana Mill. & 10 & 38.46 & $\mathrm{CP}$ & $N$ & México \\
\hline Poaceae & Zea mays L. & 10 & 38.46 & $\mathrm{C}$ & $\mathrm{N}$ & México a Guatemala \\
\hline Punicaceae & Punica granatum L. & 10 & 38.46 & $\mathrm{C}$ & I & Mediterráneo, sur de Asia \\
\hline Anacardiaceae & Spondias purpurea L. & 9 & 34.62 & SC & $\mathrm{N}$ & América tropical \\
\hline Annonaceae & Annona cherimola Mill. & 9 & 34.62 & $\mathrm{C}$ & I & Ecuador y Perú \\
\hline Fabaceae & Leucaena esculenta (DC.) Benth. & 9 & 34.62 & CPS & $\mathrm{N}$ & América \\
\hline Julianiaceae & $\begin{array}{l}\text { Amphipterygium adstringens } \\
\text { Schiede ex Schltdl. }\end{array}$ & 9 & 34.62 & SP & $\mathrm{N}$ & $\begin{array}{l}\text { Vertiente del Pacífico } \\
\text { mexicano }\end{array}$ \\
\hline Malpighiaceae & Byrsonima crassifolia (L.) Kunth & 9 & 34.62 & $\mathrm{CP}$ & $\mathrm{N}$ & América tropical \\
\hline Rutaceae & Citrus aurantium L. & 9 & 34.62 & $\mathrm{C}$ & I & Sudeste de Asia \\
\hline Sterculiaceae & Guazuma ulmifolia Lam. & 9 & 34.62 & Nat & I & Antillas \\
\hline Acanthaceae & Jacobinia spicigera (Schltdl.) L.H. Bailey & ey 8 & 30.77 & $\mathrm{C}$ & $\mathrm{N}$ & México a Colombia \\
\hline Bombacaceae & Ceiba aesculifolia (Kunth) Rose & 8 & 30.77 & SC & $\mathrm{N}$ & $\begin{array}{c}\text { América tropical y } \\
\text { subtropical }\end{array}$ \\
\hline Euphorbiaceae & Ricinus communis $\mathrm{L}$. & 8 & 30.77 & Nat & I & Africa \\
\hline Myrtaceae & Eucalyptus globulus Labill. & 8 & 30.77 & C & I & Australia \\
\hline Rosaceae & Eriobotrya japonica (Thunb.) Lindl. & 8 & 30.77 & $\mathrm{C}$ & I & China \\
\hline Arecaceae & Cocos nucifera L. & 7 & 26.92 & $\mathrm{C}$ & I & Océano Pacífico \\
\hline Asteraceae & $\begin{array}{l}\text { Artemisia ludoviciana spp. mexicana } \\
\text { (Willd.) Keck }\end{array}$ & 7 & 26.92 & $\mathrm{P}$ & $\mathrm{N}$ & $\begin{array}{c}\text { Sur de Estados Unidos a } \\
\text { Guatemala }\end{array}$ \\
\hline Asteraceae & Tagetes erecta $\mathrm{L}$. & 7 & 26.92 & $\mathrm{P}$ & $\mathrm{N}$ & México \\
\hline Ebenaceae & Diospyros digyna Jacq. & 7 & 26.92 & $\mathrm{CP}$ & $\mathrm{N}$ & México y Centroamérica \\
\hline Lamiaceae & Marrubium vulgare L. & 7 & 26.92 & $\mathrm{C}$ & I & Eurasia \\
\hline Lamiaceae & Mentha piperita L. & 7 & 26.92 & $\mathrm{C}$ & I & Europa, Asia, Africa boreal \\
\hline Rosaceae & Prunus persica (L.) Batsch & 7 & 26.92 & $\mathrm{C}$ & I & China \\
\hline Rosaceae & $\begin{array}{l}\text { Prunus serotina ssp. capuli } \\
\text { (Cav.) McVaugh }\end{array}$ & 7 & 26.92 & CPS & $\mathrm{N}$ & América boreal \\
\hline Rutaceae & Casimiroa edulis La Llave et Lex. & 7 & 26.92 & SC & $\mathrm{N}$ & México y Centroamérica \\
\hline Asteraceae & Matricaria recutita $\mathrm{L}$. & 6 & 23.08 & $\mathrm{C}$ & I & Europa \\
\hline Fabaceae & Lysiloma divaricatum (Jacq.) J.F.Macbr. & 6 & 23.08 & $\mathrm{~S}$ & $\mathrm{~N}$ & América tropical \\
\hline Malpighiaceae & Malpighia mexicana A. Juss. & 6 & 23.08 & $\mathrm{~S}$ & $\mathrm{~N}$ & México \\
\hline Nyctaginaceae & Bougainvillea glabra Choisy & 6 & 23.08 & $\mathrm{C}$ & I & Ecuador al sur de Brasil \\
\hline Poaceae & Saccharum officinarum L. & 6 & 23.08 & $\mathrm{C}$ & I & Sureste de Asia \\
\hline Sapotaceae & $\begin{array}{l}\text { Pouteria sapota (Jacq.) H.E. Moore } \\
\text { et Stearn }\end{array}$ & 6 & 23.08 & SC & $\mathrm{N}$ & Mesoamérica \\
\hline Anacardiaceae & Spondias mombin L. & 5 & 19.23 & SC & $\mathrm{N}$ & América tropical \\
\hline Apocynaceae & Nerium oleander L. & 5 & 19.23 & $\mathrm{C}$ & I & $\begin{array}{c}\text { Mediterráneo al oeste de } \\
\text { China }\end{array}$ \\
\hline Asteraceae & Sanvitalia procumbens Lam. & 5 & 19.23 & $\mathrm{P}$ & $\mathrm{N}$ & América \\
\hline Asteraceae & Tagetes lucida Cav. & 5 & 19.23 & $\mathrm{P}$ & $\mathrm{N}$ & América \\
\hline Bignoniaceae & Parmentiera aculeata (Kunth) Seem. & 5 & 19.23 & SP & $\mathrm{N}$ & América \\
\hline
\end{tabular}


Columba Monroy-Ortiz y Rafael Monroy

\begin{tabular}{|c|c|c|c|c|c|c|}
\hline \multirow[t]{2}{*}{ Familia } & \multirow[t]{2}{*}{ Nombre científico } & \multicolumn{2}{|c|}{$\begin{array}{l}\text { Dominancia cultural } \\
\text { (Citas) }\end{array}$} & \multirow[t]{2}{*}{ Manejo } & \multirow[t]{2}{*}{ Origen } & \multirow[t]{2}{*}{$\begin{array}{l}\text { Región de origen } \\
\text { o distribución }\end{array}$} \\
\hline & & No. & $\%$ & & & \\
\hline Bignoniaceae & Tecoma stans (L.) Juss. ex Kunth & 5 & 19.23 & $\mathrm{P}$ & $\mathrm{N}$ & $\begin{array}{l}\text { América tropical y } \\
\text { subtropical }\end{array}$ \\
\hline Burseraceae & Bursera copallifera (DC.) Bullock & 5 & 19.23 & SP & $\mathrm{N}$ & América \\
\hline Burseraceae & Bursera glabrifolia (Kunth) Engl. & 5 & 19.23 & SP & $\mathrm{N}$ & México y Centroamérica \\
\hline Convolvulaceae & Ipomoea murucoides Roem. et Schult. & 5 & 19.23 & $\mathrm{P}$ & $\mathrm{N}$ & México \\
\hline Euphorbiaceae & $\begin{array}{l}\text { Euphorbia pulcherrima Willd. } \\
\text { ex Klotzsch }\end{array}$ & 5 & 19.23 & $\mathrm{C}$ & $\mathrm{N}$ & $\begin{array}{l}\text { Sur de México } \\
\text { y Guatemala }\end{array}$ \\
\hline Fabaceae & Acacia farnesiana (L.) Willd. & 5 & 19.23 & $\mathrm{~S}$ & $\mathrm{~N}$ & América tropical \\
\hline Fabaceae & Delonix regia (Bojer ex Hook.) Raf. & 5 & 19.23 & CNat & I & Madagascar \\
\hline Fabaceae & $\begin{array}{l}\text { Enterolobium cyclocarpum } \\
\text { (Jacq.) Griseb. }\end{array}$ & 5 & 19.23 & $\mathrm{SP}$ & $\mathrm{N}$ & América tropical \\
\hline Fabaceae & Tamarindus indica L. & 5 & 19.23 & CNat & I & África tropical \\
\hline Lamiaceae & Ocimum basilicum L. & 5 & 19.23 & C & I & Tropico asiático y africano \\
\hline Lamiaceae & Rosmarinus officinalis $\mathrm{L}$. & 5 & 19.23 & C & I & Mediterráneo \\
\hline Oleaceae & Fraxinus uhdei (Wenzig) Lingelsh. & 5 & 19.23 & SC & $\mathrm{N}$ & $\begin{array}{l}\text { Sinaloa y Durango hasta } \\
\text { Veracruz y Chiapas }\end{array}$ \\
\hline Rosaceae & Pyrus communis $\mathrm{L}$. & 5 & 19.23 & C & 1 & Europa, Asia menor \\
\hline Rutaceae & Citrus grandis (L.) Osbeck & 5 & 19.23 & $\mathrm{C}$ & I & Asia \\
\hline Rutaceae & Citrus limon (L.) Burm. f. & 5 & 19.23 & $\mathrm{C}$ & 1 & Asia \\
\hline Rutaceae & Citrus reticulata Blanco & 5 & 19.23 & $\mathrm{C}$ & I & Sureste de Asia \\
\hline Sapotaceae & Manilkara zapota (L.) Royen & 5 & 19.23 & SC & $\mathrm{N}$ & Mesoamérica \\
\hline Verbenaceae & Lippia dulcis Trevir. & 5 & 19.23 & Nat & 1 & Cuba \\
\hline Alliaceae & Allium cepa L. & 4 & 15.38 & $\mathrm{C}$ & I & Persia \\
\hline Alliaceae & Allium sativum $\mathrm{L}$. & 4 & 15.38 & $\mathrm{C}$ & I & Asia central \\
\hline Apiaceae & Foeniculum vulgare Mill. & 4 & 15.38 & $\mathrm{C}$ & 1 & Sur y sureste de Asia \\
\hline Apocynaceae & Plumeria rubra L. & 4 & 15.38 & SC & $\mathrm{N}$ & México a Centroamérica \\
\hline Asphodelaceae & Aloe barbadensis Mill. & 4 & 15.38 & $\mathrm{C}$ & 1 & Arabia, noreste de Africa \\
\hline Asteraceae & Heterotheca inuloides Cass. & 4 & 15.38 & $\mathrm{P}$ & $\mathrm{N}$ & Norteamérica \\
\hline Begoniaceae & Begonia gracilis Kunth & 4 & 15.38 & $\mathrm{~S}$ & $\mathrm{~N}$ & México a Guatemala \\
\hline Bignoniaceae & Spathodea campanulata P. Beauv. & 4 & 15.38 & $\mathrm{C}$ & 1 & África tropical \\
\hline Cactaceae & Stenocereus stellatus (Pfeiff.) Riccob. & 4 & 15.38 & $\mathrm{~S}$ & $\mathrm{~N}$ & México \\
\hline Caprifoliaceae & $\begin{array}{l}\text { Sambucus nigra ssp. canadensis } \\
\text { (L.) R. Bolli }\end{array}$ & 4 & 15.38 & SC & $\mathrm{N}$ & Canadá a Panamá y Antillas \\
\hline Caricaceae & Jacaratia mexicana A. DC. & 4 & 15.38 & $\mathrm{~s}$ & $\mathrm{~N}$ & México a Panamá \\
\hline Cucurbitaceae & Cucurbita pepo L. & 4 & 15.38 & C & $\mathrm{N}$ & México \\
\hline Euphorbiaceae & Euphorbia prostrata Aiton & 4 & 15.38 & $\mathrm{P}$ & N & $\begin{array}{c}\text { Sudeste de Estados Unidos } \\
\text { a Sudamérica }\end{array}$ \\
\hline Fabaceae & Eysenhardtia polystachya (Ortega) Sarg. & 5. 4 & 15.38 & SP & $\mathrm{N}$ & México \\
\hline Fabaceae & Phaseolus vulgaris $\mathrm{L}$. & 4 & 15.38 & C & $\mathrm{N}$ & América \\
\hline Fabaceae & Prosopis juliflora (Sw.) DC. & 4 & 15.38 & $\mathrm{P}$ & $\mathrm{N}$ & Norte a Centroamérica \\
\hline Lamiaceae & Mentha canadensis L. & 4 & 15.38 & C & I & América boreal, China \\
\hline Lamiaceae & Mentha pulegium L. & 4 & 15.38 & $\mathrm{C}$ & I & Europa y oeste de Asia \\
\hline Lauraceae & Cinnamomum verum J. Presl & 4 & 15.38 & $\mathrm{C}$ & I & Sudeste de Asia \\
\hline Loganiaceae & Buddleia sessiliflora Kunth & 4 & 15.38 & $\mathrm{P}$ & $\mathrm{N}$ & $\begin{array}{c}\text { Baja California y Texas a } \\
\text { Oaxaca }\end{array}$ \\
\hline Malvaceae & Anoda cristata (L.) Schltdl. & 4 & 15.38 & $\mathrm{P}$ & $\mathrm{N}$ & México \\
\hline Malvaceae & Hibiscus rosa-sinensis L. & 4 & 15.38 & $\mathrm{C}$ & I & Asia tropical \\
\hline Meliaceae & Trichilia hirta L. & 4 & 15.38 & SP & $\mathrm{N}$ & América tropical \\
\hline Moraceae & Ficus petiolaris Kunth & 4 & 15.38 & $\mathrm{~S}$ & $\mathrm{~N}$ & México \\
\hline Pinaceae & Pinus montezumae Lamb. & 4 & 15.38 & $\mathrm{~S}$ & $\mathrm{~N}$ & México \\
\hline Plantaginaceae & Plantago major $\mathrm{L}$. & 4 & 15.38 & Nat & I & Viejo mundo \\
\hline Rosaceae & Cydonia oblonga Mill. & 4 & 15.38 & $\mathrm{C}$ & 1 & Irán a Turkestán \\
\hline
\end{tabular}


DominanCia CUltural de las Plantas Útiles DE Morelos

\begin{tabular}{|c|c|c|c|c|c|c|}
\hline \multirow[t]{2}{*}{ Familia } & \multirow[t]{2}{*}{ Nombre científico } & \multicolumn{2}{|c|}{$\begin{array}{l}\text { Dominancia cultural } \\
\text { (Citas) }\end{array}$} & \multirow[t]{2}{*}{ Manejo } & \multirow[t]{2}{*}{ Origen } & \multirow[t]{2}{*}{$\begin{array}{l}\text { Región de origen } \\
\text { o distribución }\end{array}$} \\
\hline & & No. & $\%$ & & & \\
\hline Rosaceae & Malus pumila Mill. & 4 & 15.38 & C & I & Europa, Asia menor \\
\hline Rosaceae & Rosa gallica var. centifolia (L.) Regel & 4 & 15.38 & $\mathrm{C}$ & I & Europa, Asia \\
\hline Rubiaceae & Coffea arabica L. & 4 & 15.38 & C & 1 & Etiopia \\
\hline Rubiaceae & $\begin{array}{l}\text { Randia echinocarpa Moc. et } \\
\text { Sessé ex DC. }\end{array}$ & 4 & 15.38 & $\mathrm{P}$ & $\mathrm{N}$ & México \\
\hline Rutaceae & Citrus limetta Risso & 4 & 15.38 & $\mathrm{C}$ & I & Asia tropical \\
\hline Rutaceae & Citrus sinensis (L.) Osbeck & 4 & 15.38 & C & 1 & Sur de China y Vietnam \\
\hline Sapindaceae & Dodonaea viscosa (L.) Jacq. & 4 & 15.38 & $\mathrm{P}$ & $\mathrm{N}$ & América \\
\hline Solanaceae & Brugmansia candida Pers. & 4 & 15.38 & C & 1 & Sudamérica \\
\hline Solanaceae & Brugmansia $\times$ candida Pers. & 4 & 15.38 & CNat & I & Ecuador \\
\hline Solanaceae & Lycopersicon esculentum Mill. & 4 & 15.38 & C & 1 & Perú a Ecuador \\
\hline Solanaceae & Physalis philadelphica Lam. & 4 & 15.38 & $\mathrm{CP}$ & $\mathrm{N}$ & México \\
\hline Solanaceae & Solanum nigrum L. & 4 & 15.38 & Nat & 1 & Eurasia \\
\hline Verbenaceae & Lantana camara L. & 4 & 15.38 & SC & $\mathrm{N}$ & América tropical \\
\hline Verbenaceae & Vitex mollis Kunth & 4 & 15.38 & SP & $\mathrm{N}$ & $\begin{array}{l}\text { Vertiente pacífica de } \\
\text { México }\end{array}$ \\
\hline
\end{tabular}

$\mathrm{C}=$ Cultivada; Nat = Naturalizada; $\mathrm{P}=$ crece en áreas perturbadas $; \mathrm{S}=$ silvestre (crece en zonas conservadas) $\mathrm{I}=\mathrm{Introducida} ;$ $\mathrm{N}=$ Nativa. 